\title{
Numerical Issues for Circulation Control Calculations
}

\author{
R. C. Swanson* and C. L. Rumsey ${ }^{\dagger}$ \\ NASA Langley Research Center, Hampton, VA 23681-2199, USA
}

\begin{abstract}
Steady-state and time-accurate two-dimensional solutions of the compressible Reynolds-averaged NavierStokes equations are obtained for flow over the Lockheed circulation control (CC) airfoil and the General Aviation CC (GACC) airfoil. Numerical issues in computing circulation control flows such as the effects of grid resolution, boundary and initial conditions, and unsteadiness are addressed. For the Lockheed CC airfoil computed solutions are compared with detailed experimental data, which include velocity and Reynolds stress profiles. Three turbulence models, having either one or two transport equations, are considered. Solutions are obtained on a sequence of meshes, with mesh refinement primarily concentrated on the airfoil circular trailing edge. Several effects related to mesh refinement are identified. For example, sometimes sufficient mesh resolution can exclude nonphysical solutions, which can occur in CC airfoil calculations. Also, sensitivities of the turbulence models with mesh refinement are discussed. In the case of the GACC airfoil the focus is on the difference between steady-state and time-accurate solutions. A specific objective is to determine if there is self-excited vortex shedding from the jet slot lip.
\end{abstract}

\section{Introduction}

Computational methods will play a vital role in designing effective circulation control (CC) configurations. Certainly, detailed experimental data, such as velocity profiles and Reynolds stresses, will be absolutely essential for validating these prediction tools. Due to the cost of flow control experiments, design and parametric studies will strongly depend on accurate and efficient prediction methods. These methods must have the potential to treat pulsating jets, even multiple jets, for a broad range of flow conditions (e.g., Mach number, Reynolds number, angle of attack). In general, the numerical methods must be extendable to time-dependent and three-dimensional flows. Furthermore, the process of developing these methods necessarily demands careful exploration of computational issues such as numerical accuracy, boundary and initial conditions, and appropriately resolving unsteady behavior. The various aspects of turbulence modeling such as numerical approximations and modeling of the terms appearing in transport equations must also be adequately understood.

At the circulation control workshop ${ }^{1}$ held at NASA Langley in 2004, an effort was made to assess the capability at that time to calculate CC airfoil flows. Although the assessment included a wide range of applications, the focus was the computation of the flow over the $\mathrm{CC}$ airfoil configuration with a circular trailing edge (NCCR 1510-7067) tested by Abramson. ${ }^{2}$ Various computational methods and a variety of turbulence models, ranging from an algebraic model to a full Reynolds stress model, were considered. One of the conclusions that can be drawn from this workshop is that turbulence modeling is a crucial element in establishing accurate prediction methods. The reliability of the models depends on their capability to consistently predict the location of the jet detachment from the Coanda surface. Since the accuracy of the numerical solution is inextricably linked to the turbulence modeling, there can be significant sensitivity to mesh effects. Of course, the sensitivity depends on the degree of stiffness of the turbulence transport equation or equations as well as the approximations due to both discretization and modeling of specific terms (e.g., advection, production, dissipation). When computing flow solutions involving multiple viscous shear layers (i.e., wall boundary layer, jet, mixing region) this sensitivity appears to be even greater.

\footnotetext{
* Senior Research Scientist, Computational AeroSciences Branch, Mail Stop 128, Senior Member AIAA.

†Senior Research Scientist, Computational AeroSciences Branch, Mail Stop 128, Associate Fellow AIAA.

This material is declared a work of the U.S. Government and is not subject to copyright protection in the United States.2006
} 
In this paper we consider several numerical issues when computing $\mathrm{CC}$ airfoil flows. These issues can be divided into two general areas. The first area is mesh resolution. It is well known that modeling turbulence with one or more transport equations introduces a more demanding requirement for sufficiently fine mesh spacing near solid boundaries (i.e., that the first mesh point off the boundary is inside the laminar sublayer). In the case of a $\mathrm{CC}$ airfoil with a rounded trailing edge there can also be a more stringent resolution requirement in the tangential (streamwise) direction along the Coanda surface. The failure to have adequate streamwise resolution can result in nonphysical solutions such as those seen in Swanson et al. ${ }^{3}$ While insufficient mesh resolution may not be the only factor producing such solutions, it is certainly an important one.

The second computational area that we address is the time-dependent iterative process that is frequently used to solve fluid dynamic equations, including turbulence transport equations. For stationary (steadystate) calculations the assumption is the following: If a steady-state solution exists, then starting with an arbitrary initial solution the iterative numerical scheme will converge to a unique steady-state solution. There is also the situation where oscillations in global quantities, such as lift and drag coefficients, appear in steady-state calculations involving turbulence transport equations. Such behavior frequently occurs, and it at least suggests that the flow may be unsteady. However, in the present work we show examples where the oscillations completely disappear when a time-accurate computation is performed. In addition, we also present a solution computed time accurately for a CC airfoil flow that is significantly different from the near stationary solution (i.e., oscillations occur in the aerodynamic coefficients) computed without time accuracy. Baker $^{4}$ has also observed significant differences between near steady-state solutions and the corresponding time-accurate solutions for flow over the General Aviation Circulation Control (GACC) airfoil.

From the results of Baker a number of questions have also emerged related to computational issues for $\mathrm{CC}$ airfoil flow simulations. In Baker's work the incompressible Reynolds-averaged Navier-Stokes (RANS) equations were solved for flow over the GACC airfoil. The Mach number was about 0.1, the angle of attack was zero degrees, and the Reynolds number based on chord was $0.533 \times 10^{6}$. Several values of the jet momentum coefficient $C_{\mu}$ (ratio of jet momentum to free-stream momentum) were considered. For these applications the grid was highly concentrated in the vicinity of the main jet slot and its lip. At $C_{\mu}$ above approximately 0.4 , steady-state calculations indicated possible unsteadiness in the flow. In addition, the jet flow separated too late, adhering to the Coanda surface and continuing onto the lower surface of the airfoil. By computing these flows time accurately, Baker obtained unsteady solutions, with high-frequency vortex shedding from the lip of the jet slot. Also, the jet flow separated on the Coanda surface rather than proceeding onto the lower airfoil surface. Two primary interesting questions arise concerning these results. First, what are the effects of computing a flow as incompressible when there are highly compressible regions in the flow? In the vicinity of the jet slot the Mach number can be transonic and the density of the flow can be reduced to about 0.6 of the free-stream density. Second, what is the effect of specifying the jet velocity at the jet exit plane rather than computing it by including the plenum flow? For the calculations of Baker the jet velocity was specified at the exit plane for computational efficiency. In performing similar calculations we have not observed the unsteady behavior reported by Baker.

In this paper we address the aforementioned numerical issues related to mesh resolution and timedependent computations. A sequence of two-dimensional (2-D) meshes, with the finest mesh exceeding 450, 000 points, is used to determine mesh effects. Computational results are compared to the detailed experimental data of Novak. ${ }^{5}$ Since various turbulence models respond differently to changes in mesh resolution, we consider three representative turbulence models.

With respect to time-dependent computations we present circumstances for the occurrence of nonunique solutions. Furthermore, we show a situation where different solutions are obtained depending upon the iterative path. In the final section of the paper the questions concerning the GACC airfoil calculations of Baker are addressed. The issues that are derived from these questions have far greater implications than just what is occurring in one set of simulations.

\section{Geometry and Flow Conditions}

The circulation control wing $(\mathrm{CCW})$ model in the Novak et al. experiment has a chord of 15 inches and a span of 24 inches, resulting in an aspect ratio of 1.6. The airfoil section has a supercritical leading edge, symmetric middle section and a 2 inch diameter Coanda CC aft section. It has a thickness ratio of $15.6 \%$. The jet slot height-to-chord ratio is 0.002 , which corresponds to a slot height of 0.03 inches. 


\begin{tabular}{|c|r|}
\hline$C_{\mu}$ & $\alpha_{\text {cor }}(\mathrm{deg})$ \\
\hline 0.030 & -2.46 \\
0.100 & -5.86 \\
0.226 & -8.94 \\
\hline
\end{tabular}

Table 1. Angle of attack corrections for Lockheed CC airfoil.

For the tests conducted by Novak et al. the free-stream Mach number is 0.12 and the Reynolds number is $0.986 \times 10^{6}$. The geometric angle of attack is zero degrees. The values of the jet momentum coefficient $\left(C_{\mu}\right)$, which is the ratio of the jet momentum to the free-stream momentum, are 0.03 and 0.10 . The measured data include not only surface pressure distributions, but also mean flow velocity profiles and Reynolds stress profiles taken using Laser doppler velocimetry (LDV). This includes data on the Coanda surface. In this paper we consider only the $C_{\mu}=0.10$ case.

A 2-D circulation control wing model with a GACC airfoil section was tested by Jones et al. ${ }^{6}$ The GACC airfoil is a $17 \%$ supercritical airfoil. The chord and span of the wing are 9.40 inches and 28.0 inches, respectively. The model contains two jet slots, one located on the upper surface at $x / c=0.985$ and the other on the bottom surface at $x / c=0.975$. Slot heights nondimensionalized by the chord range from 0.0011 to 0.0021 (0.01 inches to 0.02 inches). Three different trailing edge configurations can be accomodated with the model. In this work we consider the circular trailing edge having a radius to chord ratio of 0.02 and a slot height of 0.01 inches.

For the tests conducted by Jones et al. the free-stream Mach number is 0.10 and the Reynolds number is $0.533 \times 10^{6}$. The geometric angle of attack is zero degrees. The values of the jet momentum coefficient $C_{\mu}$ vary from 0.013 to about 0.200 .

Although these experiments are designed to be two-dimensional, there are three-dimensional effects due to the presence of the sidewalls in the wind tunnel. The interaction of the sidewall boundary layers with the wing and its Coanda surface produce vortical structures that induce a downwash along the span of the wing, reducing the effective angle of attack. For the Lockheed CC airfoil the angle of attack correction suggested by the experimenters is given in Table 1. Note that these corrections are rather large, due in part to the significant influence of the side-wall interactions. For the GACC airfoil there are no angle of attack corrections provided by the experimenters.

\section{Computational Grids}

In order to assess the effects of mesh resolution a series of grids were generated for the Lockheed CC airfoil case being considered. Primarily, increased resolution is focused on the circular trailing edge (Coanda surface). The variation in total and trailing edge grid densities is given in Table 2. Minimum normal grid spacing and the typical grid spacing in the tangential (streamwise) direction are also included in the table. All grids are defined with six blocks except grid 2. Grid 2, which is defined by three blocks, is the same grid used for the computational results presented in Ref. 3. The domain is decomposed in the following manner. There are one block for the leading edge section of the airfoil, two blocks for the lower and upper middle sections, two blocks on the circular trailing edge, and one block for the plenum. For Grids $4-7$ there are 632 points around the airfoil, excluding those on the Coanda surface, and there are $161 \times 81$ points in the plenum. Both the normal and tangential spacings are a factor of five smaller for the finest grid (grid 7) than grid 2. For grid 2 the spacing immediately behind the lip of the jet slot is $1.0 \times 10^{-3}$ chords, and the lip is treated as an inviscid surface. The spacing behind the lip for all other grids is between $1.0 \times 10^{-5}$ and $2.5 \times 10^{-5}$ chords, and the lip is treated as a viscous surface. For all grids there is tangential clustering of the grid at the airfoil leading edge, and the outer boundary is always located 40 chords away from the airfoil surface. "Medium" and "coarse" versions of each "fine" grid are generated by eliminating every other grid line in each coordinate direction.

For reference, Lockheed grid 4 is shown in Fig. 1. Figures 2 and 3 illustrate the resolution used for two of the Lockheed grids, grid 4 and grid 5, in the region at the start of the Coanda surface immediately aft of the slot exit. In these latter two figures, the slot is shown at the lower left; above this is the (blunt) slot 


\begin{tabular}{|c|c|c|c|c|c|}
\hline $\begin{array}{c}\text { Airfoil } \\
\text { Geometry }\end{array}$ & $\begin{array}{c}\text { Grid } \\
\text { Number }\end{array}$ & $\begin{array}{c}\text { Total Grid } \\
\text { Density }\end{array}$ & $\begin{array}{c}\text { Circular TE } \\
\text { Grid Density }\end{array}$ & $\begin{array}{c}(\Delta y)_{\min } / c \\
\text { on TE }\end{array}$ & $\begin{array}{c}\Delta s / c \\
\text { on TE }\end{array}$ \\
\hline Lockheed & 2 & 114,336 & $181 \times 241$ & $1.0 \times 10^{-5}$ & $1.0 \times 10^{-3}$ \\
& 4 & 266,944 & $425 \times 289$ & $1.0 \times 10^{-5}$ & $4.0 \times 10^{-4}$ \\
& 5 & 303,936 & $553 \times 289$ & $1.0 \times 10^{-5}$ & $4.0 \times 10^{-4}$ \\
& 6 & 303,936 & $553 \times 289$ & $2.0 \times 10^{-6}$ & $4.0 \times 10^{-4}$ \\
& 7 & 463,464 & $1105 \times 289$ & $2.0 \times 10^{-6}$ & $2.0 \times 10^{-4}$ \\
\hline GACC & 1 & 278,528 & $625 \times 193$ & $1.0 \times 10^{-6}$ & $4.0 \times 10^{-6}$ (slot) \\
& & & & & $6.0 \times 10^{-4}$ \\
& 2 & 434,896 & $1089 \times 193$ & $1.0 \times 10^{-6}$ & $4.0 \times 10^{-6}$ (slot) \\
& & & & & $8.0 \times 10^{-5}$ \\
\hline
\end{tabular}

Table 2. Characteristics of fine grids used for CC airfoil calculations. All $\Delta s / c$ values are typical unless otherwise noted.

upper lip.

For the GACC airfoil case, block structured grids were also generated. Three blocks were used to partition the discretized domain. One block is in the plenum, another one is adjacent to the Coanda surface, and the remaining block surrounds the entire airfoil. The grid 1 for the calculations includes $129 \times 97$ points in the plenum, 625 points along the Coanda surface and 193 points normal to it, 1008 points around the airfoil (excluding those on the Coanda surface) and 145 points in the radial direction. Coarser versions of a given grid are created in the same manner as for the Lockheed grids.

The grid, as seen in Fig. 4, is clustered near the surface in order to resolve the turbulent boundary layers. Normal grid spacing adjacent to the surface for the fine grid is about $1 \times 10^{-6}$ chords. In the vicinity of the lip of the upper surface jet slot the grid is highly clustered (see Fig. 5), so as to resolve the vortices behind the lip and capture possible unsteadiness due to vortex shedding.

\section{Numerical Method}

Numerical solutions were computed with CFL3D, a multi-zone compressible RANS code developed at NASA Langley. ${ }^{7}$ It solves the thin-layer form of the Navier-Stokes equations in each of the (selected) coordinate directions. It can use one-to-one, patched, or overset grids, and employs local time step scaling, grid sequencing, and multigrid to accelerate convergence to steady state. In time-accurate mode, CFL3D has the option to employ dual-time stepping with subiterations and multigrid, and it achieves second-order temporal accuracy.

The code CFL3D is based on a finite-volume method. The convective and pressure terms are approximated with third-order upwind-biased spatial differencing, and the viscous terms are discretized with second-order central differencing. The discrete scheme is globally second-order spatially accurate. The flux difference-splitting (FDS) method $^{8}$ of Roe is employed to obtain fluxes at the cell faces. Advancement in time is accomplished with an implicit approximate factorization method (number of factors determined by number of dimensions).

In CFL3D, the turbulence models are implemented uncoupled from the mean-flow equations. The turbulent transport equations are solved using an implicit approximate factorization approach. The advection terms are discretized with either first-order or second-order upwind differencing, with first-order the default. The production source term is treated explicitly, while the advection, destruction, and diffusion terms are treated implicitly. 


\section{Boundary and Initial Conditions}

Boundary conditions are required at the inflow (internal and external), outflow, and solid surface boundaries. For numerical computations the physical boundary conditions must be supplemented with numerical boundary conditions, which generally involve extrapolation of flow quantities or combinations of them (e.g., Riemann invariants) from the interior of the domain. Discussion of the numerical boundary conditions is given in the user's manual for CFL3D. ${ }^{7}$ At the far-field inflow boundary a Riemann invariant, entropy, and flow inclination angle are specified. A Riemann invariant is specified at the far-field outflow boundary. At the upstream wall of the plenum the velocity and density are specified such that the desired $C_{\mu}$ is obtained. At the surface boundaries the no-slip and adiabatic wall conditions are specified. Boundary conditions for the various turbulence models considered herein are given in Ref. 7 . The initial solution is defined by the free-stream conditions, or a coarser grid solution, or a previous solution.

\section{Turbulence Modeling}

Unless otherwise indicated, all the CC airfoil flow computations were performed fully turbulent; three turbulence models are considered. One is the one-equation Spalart-Allmaras (SA) model ${ }^{9}$ with rotation/curvature (SARC) modification. ${ }^{10,11}$ Another model is the two-equation shear-stress transport (SST) model of Menter. ${ }^{12-14}$ The third is the $k-\zeta$ model,${ }^{15}$ which is a two-equation model for the turbulent kinetic energy and the enstrophy. The enstrophy represents the variance of vorticity. All of these models are linear eddy-viscosity models that make use of the Boussinesq eddy-viscosity hypothesis. The equations describing these three models can be found in their respective references. However, there are certain details concerning the implementation of the SARC and SST models that are given here in order to precisely identify what form of the model is being applied.

The SA model can be written in general form as

$$
\frac{D \tilde{\nu}}{D t}=\mathcal{P}+\mathcal{D}_{\text {diff }}+\mathcal{D}_{\text {diss }}
$$

where $\tilde{\nu} \sim \nu_{t}$, and $\mathcal{P}, \mathcal{D}_{\text {diff }}$, and $\mathcal{D}_{\text {diss }}$ are the contributions associated with turbulence due to production, diffusion, and dissipation, respectively. The production term is given by

$$
\mathcal{P}=c_{b 1}\left[1-f_{t 2}\right] W \tilde{\nu} .
$$

In the SARC model $\mathcal{P}$ is replaced by

$$
\begin{gathered}
\mathcal{P}^{\prime}=c_{b 1}\left[f_{r 1}-f_{t 2}\right] W \tilde{\nu}, \\
f_{r 1}=\left(1+c_{r 1}\right) \frac{2 r^{*}}{\left(1+r^{*}\right)}\left[1-c_{r 3} \tan ^{-1}\left(c_{r 2} \tilde{r}\right)\right]-c_{r 1},
\end{gathered}
$$

where the function $r^{*}$ is the ratio of scalar measure of strain rate to the scalar measure of rotation, the function $\tilde{r}$ depends on the Lagrangian derivative of the strain-rate tensor principal axes angle (see Ref. 11 for details), and $c_{r 1}=1, c_{r 2}=12$, and $c_{r 3}=0.6-1.0$. As $c_{r 3}$ is increased, the turbulence production will decrease near convex surfaces. In the results for this paper we use $c_{r 3}=1.0$.

The production term $\mathcal{P}_{k}$ in the turbulent kinetic energy equation of the Menter SST model can be written as

$$
\mathcal{P}_{k}=\tau_{i j} \frac{\partial u_{i}}{\partial x_{j}}
$$

where the stress tensor $\tau_{i j}$ is defined as

$$
\tau_{i j}=\mu_{t}\left(\frac{\partial u_{i}}{\partial x_{j}}+\frac{\partial u_{j}}{\partial x_{i}}-\frac{2}{3} \frac{\partial u_{m}}{\partial x_{m}} \delta_{i j}\right)-\frac{2}{3} \rho k \delta_{i j},
$$

and $\mu_{t}$ is the turbulent viscosity, the partial derivatives are strain rates, and $k$ is the turbulent kinetic energy. The production term $\mathcal{P}_{\omega}$ in the $\omega$ equation of the SST model is proportional to $\mathcal{P}_{k}$. Generally, in the computations with the SST model, the incompressible assumption is imposed, and the turbulent kinetic energy contribution is neglected. Thus,

$$
\mathcal{P}_{k} \approx \mu_{t}\left(\frac{\partial u_{i}}{\partial x_{j}}+\frac{\partial u_{j}}{\partial x_{i}}\right) \frac{\partial u_{i}}{\partial x_{j}}=2 \mu_{t} S_{i j} S_{i j}
$$


where $S_{i j}$ is the strain-rate tensor, and $S_{i j} S_{i j}$ represents the dot product of two tensors. Note that it is also common as a further approximation to employ the vorticity rather than the strain-rate tensor (see Menter ${ }^{12}$ ). The use of vorticity in the production term was found in Swanson et al. ${ }^{16}$ to have a greater tendency to produce unphysical jet wraparound onto the lower surface. In the present work we use Eq. (7) for $\mathcal{P}_{k}$.

The eddy viscosity determined with the SST model is defined as

$$
\nu_{t}=\frac{a_{1} k}{\max \left(a_{1} \omega ; \Omega F_{2}\right)},
$$

where $a_{1}$ is a constant, $\omega$ is equal to the ratio of the turbulent dissipation rate to the turbulent kinetic energy, $\Omega=\sqrt{2 W_{i j} W_{i j}}$ (magnitude of the vorticity vector), and $F_{2}$ is a blending function.

\section{Numerical Results}

This section is divided into two parts. In the first part we focus on the particular numerical issues of mesh refinement and time iteration effects. The Lockheed CC airfoil is used for this purpose, since detailed experimental data is available. In the second part we consider the GACC airfoil, and the emphasis is placed on solution effects due to time-accurate computation. We address the question of possible self-excited vortex shedding from the jet slot lip. This is done both from a numerical and theoretical viewpoint. At the same time, we compare the present results with the behavior of the incompressible RANS results obtained by Baker $^{4}$ for essentially the same problem.

\section{Lockheed CC Airfoil}

The computational method described previously was first applied to the $C_{\mu}=0.10$ case from the Novak experiment. As stated earlier, and repeated here for convenience, the Mach number for the experiment is 0.12 , and the Reynolds number is $0.986 \times 10^{6}$. The corrected angle of attack is $-5.86^{\circ}$. In this section comparisons with the experimental streamline pattern as well as velocity and shear stress profiles are made. Computed and experimental pressure distributions are also compared.

\section{Mesh Refinement Effects}

As a reference we selected the solution computed with the SARC turbulence model, since this model provided the best overall comparison with the data among all the models we tested in Ref. 3. Although not included in Ref. 3, we also applied the explicit algebraic stress model (EASM) in $k-\omega$ form (EASM-ko) ${ }^{17}$ to the Lockheed CC airfoil case with $C_{\mu}=0.10$. The SARC model still provided a significantly better comparison with the experimental data. Before proceeding we first evaluate the SARC model on highly refined meshes, so as to determine its mesh sensitivity, and thus, allow an appropriate comparison with the SST and $k-\zeta$ models.

Figure 6 shows the calculated surface pressure distributions for the coarse, medium (denoted by med), and fine meshes derived from grid 7. Starting with grid 7 as the fine mesh, successive grid coarsening was done by eliminating every other mesh line in each coordinate direction. All grid levels yield oscillatory behavior on the Coanda surface immediately behind the slot exit, due in part to use of measured coordinates for the definition of the airfoil geometry but also influenced by vortical structures that occur behind the slot lip. It is interesting that to plotting accuracy the computed surface pressures on the three grid levels are nearly identical, and they generally agree quite well with the data. However, the predicted pressures are slightly lower than the measured pressures on the upper surface of the airfoil when $0.8<x / c<0.9$ and also at the trailing edge suction peak. These differences are reflected in Table 3, which presents the computed lift and drag coefficients $\left(C_{L}\right.$ and $\left.C_{D}\right)$. The experimental $C_{L}$ is 3.58 ; and thus, the computed $C_{L}$ is about $8 \%$ higher using grid 7 . Note that the drag coefficient $C_{D}$ does not include the propulsion contribution produced by the jet. There is also very good agreement between the streamlines predicted on the fine grid and those from the experiment, as displayed in Fig. 7 . In the experiment the jet flow separates at the angular location $\theta \approx 115^{\circ}$. Here, and in subsequent discussion, the angle $\theta$ is being referenced to the beginning of the circular trailing edge on the upper surface of the airfoil. For the computed flow on grid 7 , jet separation occurs at a $\theta$ of about $113^{\circ}$.

In Figs. 8a-8c the computed and experimental velocity (component parallel to surface) profiles are compared. The quantity $d$ represents distance normal to the surface. Since the jet velocity was measured, the boundary condition in the plenum was adjusted in order to match the jet velocity. Nevertheless, the 


\begin{tabular}{|c|r|c|c|}
\hline $\begin{array}{c}\text { Grid } \\
\text { Number }\end{array}$ & $\begin{array}{c}\text { Grid } \\
\text { Level }\end{array}$ & $C_{L}$ & $C_{D}$ \\
\hline 5 & coarse & 3.51 & 0.0458 \\
5 & medium & 3.80 & 0.0376 \\
5 & fine & 3.83 & 0.0443 \\
\hline 7 & coarse & 3.85 & 0.0483 \\
7 & medium & 3.87 & 0.0446 \\
7 & fine & 3.86 & 0.0436 \\
\hline
\end{tabular}

Table 3. Lift and drag coefficients computed with the SARC model for the Lockheed CC airfoil tested by Novak $\left(C_{\mu}=0.10,\left(C_{L}\right)_{\exp }=3.58\right)$.

computed velocity profiles on the initial part $\left(\theta<90^{\circ}\right)$ of the Coanda surface overpredict the measured ones. This disagreement reflects the overprediction of the experimental boundary-layer profile upstream of the jet exit (see Ref. 3). However, there is fairly good agreement with the measured data in the region of the jet separation location. To further characterize the flow we consider a comparison of the computed and experimental Reynolds stress profiles, which is presented in Figs. 9a-9c. The Reynolds stress profiles are scaled by the negative mean density and its components are aligned parallel and normal to the local surface; this was done so that we could compare directly with the tabulated measured data. In this paper we loosely refer to the scaled Reynolds stress as the Reynolds stress or shear stress. Over the initial part $\left(\theta<90^{\circ}\right)$ of the Coanda surface the predicted maximum Reynolds stress is higher than that of the experiment, while over the latter part $\left(\theta>90^{\circ}\right)$ the predicted maximum is lower than the measured one.

With the SARC model we have not observed any special sensitivity to mesh or irregular behavior with mesh refinement. However, as pointed out by Swanson, Rumsey, and Anders, ${ }^{16}$ this model does not always produce satisfactory results (i.e., good comparisons with experimental data) with the standard value of the curvature correction parameter $c_{r 3}$, which affects turbulence production. In addition, the SARC model has been applied to a relatively small number of two-dimensional problems. The SST model, on the other hand, has provided reasonably good comparisons with experimental data for a wider range of applications. Moreover, with this model we have obtained fairly good comparisons with experimental surface pressure data (see Ref. 16) for the CC airfoil tested by Abramson and Rogers. ${ }^{18}$ The SST model does, however, have much higher sensitivity to grid than the SARC model. As we demonstrate subsequently, such grid sensitivity can create numerical issues when computing CC airfoil flows.

In Ref. 3 we calculated a solution for the Lockheed CC airfoil on the medium version of grid 2 with the SST model. Figure 10a shows streamlines for that solution. They exhibit a highly nonphysical behavior, forming a large vortex below the lower surface of the airfoil. Instead of the jet detaching from the Coanda surface, it travels forward on the lower surface to almost the $x / c=0.25$ location before it turns and proceeds downstream. Steady-state convergence was not attained for this calculation, with the lift coefficient oscillating between 3.5 and 4.5. Thus, the streamlines in Fig. 10a only characterize the solution at one time during the oscillation. It should be mentioned that a similar jet behavior also occurs on both the coarse and fine versions of grid 2. As demonstrated in Refs. 3 and 19 such jet behavior is not peculiar to the SST model; it occurs for other models as well. Furthermore, solutions have been obtained where the jet not only moves upstream along the lower surface but also wraps around the airfoil.

One consideration concerning solutions with nonphysical behavior of the jet is the effect of mesh refinement, especially in the streamwise direction along the Coanda surface. Figure 10b displays the streamlines from a calculation on grid 5 using the SST model. Grid 5 has about three times as many grid points on the Coanda surface in the streamwise direction as grid 2. As revealed in the figure, on the finer grid the jet no longer traverses onto the lower surface of the airfoil. However, there is a small separation bubble on the lower surface of the airfoil near the trailing edge. This separation region generally occurs when the jet detachment is delayed much beyond $\theta=110^{\circ}-115^{\circ}$ on the Coanda surface. Although not shown, it should be noted that streamlines on grids 4 and 6 are essentially the same as those on grid 5 .

Surface pressure distributions computed on grids $4-7$ with the SST model are shown in Fig. 11. For 


\begin{tabular}{|c|r|c|c|r|c|}
\hline $\begin{array}{c}\text { Grid } \\
\text { Number }\end{array}$ & \multicolumn{1}{c|}{$\begin{array}{c}\text { Grid } \\
\text { Level }\end{array}$} & $\begin{array}{c}\text { Range of } \\
C_{L}\end{array}$ & $\begin{array}{c}\text { Range of } \\
C_{D}\end{array}$ & $\begin{array}{c}\text { Average } \\
C_{L}\end{array}$ & $\begin{array}{c}\text { Average } \\
C_{D}\end{array}$ \\
\hline 4 & coarse & $3.70-3.73$ & $0.0438-0.0753$ & 3.72 & 0.0596 \\
4 & medium & $3.81-3.85$ & $0.0490-0.0765$ & 3.83 & 0.0628 \\
4 & fine & $3.82-3.84$ & $0.0412-0.0438$ & 3.83 & 0.0425 \\
\hline 5 & medium & $3.30-3.33$ & $0.0285-0.0622$ & 3.32 & 0.0454 \\
5 & fine & $4.10-4.10$ & $0.0462-0.0462$ & 4.10 & 0.0462 \\
\hline 6 & medium & $3.60-3.63$ & $0.0537-0.0738$ & 3.62 & 0.0638 \\
6 & fine & $3.95-3.95$ & $0.0455-0.0455$ & 3.95 & 0.0455 \\
\hline 7 & coarse & $3.12-3.13$ & $0.0333-0.0400$ & 3.13 & 0.0367 \\
7 & medium & $3.13-3.14$ & $0.0378-0.0552$ & 3.14 & 0.0465 \\
7 & fine & $2.72-2.76$ & $0.0250-0.0334$ & 2.74 & 0.0332 \\
\hline
\end{tabular}

Table 4. Lift and drag coefficients computed with the SST model for the Lockheed CC airfoil tested by Novak $\left(C_{\mu}=0.10,\left(C_{L}\right)_{\exp }=3.58\right)$.

grids 4-6 the results are similar, with the pressures calculated on the upper surface somewhat lower than the measured ones. Table 4 lists the computed results. Note that for all SST steady-state computations, convergence was not achieved, although lift and drag eventually reached a quasi-steady state with oscillations. For each of the predicted aerodynamic coefficients both a range of values and an average value are given. The variation in $C_{L}$ is quite small, usually $1 \%$ or less. On the various grids a significant oscillation in the low magnitude $C_{D}$ often occurs. Of particular interest is the unexpected decrease in $C_{L}$ on grid 7 . As seen in Fig. 11, for grid 7 there is a substantial change in the surface pressures relative to those on the other grids. This difference appears to be a function of the grid distribution, and not the total number of grid points. For example, although not shown, grid 7 (med) yields surface pressure coefficients very similar to grid 7 (fine), even though grid 7 (med) has less total grid points than the fine levels of grids 4 , 5, or 6 . The pressure distribution change using grid 7 is a consequence of much earlier jet separation. These anomalous results will be discussed further below.

In Figs. 12a-12c velocity profiles computed with the SST model on the fine levels of grids $4-6$ are presented. The profiles on the various grids are quite similar. Since the jet detachment is later than that of the experiment, the discrepancies with the measured velocity profiles are much greater in the region surrounding detachment than seen with the SARC model. For the Reynolds stress profiles, which are shown in Figs. 13a-13c, the computed profiles on the set of grids is nearly the same, with the greatest differences occurring at the $\theta=110^{\circ}$ and $\theta=120^{\circ}$ locations. The stresses obtained with the SST model generally have a similar comparison with the data as those calculated with the SARC model.

Since the SST model has two transport equations and the SARC model has only one, the following question arises. Is the solution sensitivity due to mesh refinement in steady-state calculations something that occurs with all two equation models? Thus, we decided to determine what happens with the twoequation $k-\zeta$ model when the mesh is refined. In Fig. 14 the surface pressures computed on grids 4, 5, and 7 with the $k-\zeta$ model are presented. Results using this model also did not converge completely, but did reach a quasi-steady state. Unlike those with the SST model, the pressure distributions on the different grids are almost the same. The corresponding aerodynamic coefficients are given in Table 5. Strong variation in the pressures at the jet lip reflects the somewhat oscillating behavior of the solution over the initial part of the Coanda surface. This behavior in the solution can readily be discerned in the velocity profile at $\theta=30^{\circ}$. The computed streamlines (on grid 7) are compared with the experimental ones in Fig. 15. Note that there is no small separation region on the airfoil lower surface because separation occurs earlier than for the SST model, in better agreement with experiment. The velocity profiles on grids 5 and 7 when using the $k-\zeta$ model are shown in Figs. 16a-16c, and they are compared with the data as well as the profiles when using the SST model on grid 5 . The $k-\zeta$ model results generally exhibit a much better agreement with the data 


\begin{tabular}{|c|c|c|c|r|c|}
\hline $\begin{array}{c}\text { Grid } \\
\text { Number }\end{array}$ & $\begin{array}{c}\text { Grid } \\
\text { Level }\end{array}$ & $\begin{array}{c}\text { Range of } \\
C_{L}\end{array}$ & $\begin{array}{c}\text { Range of } \\
C_{D}\end{array}$ & $\begin{array}{c}\text { Average } \\
C_{L}\end{array}$ & $\begin{array}{c}\text { Average } \\
C_{D}\end{array}$ \\
\hline 4 & fine & $3.47-3.50$ & $0.0438-0.0513$ & 3.49 & 0.0476 \\
5 & fine & $3.30-3.32$ & $0.0422-0.0622$ & 3.31 & 0.0460 \\
7 & fine & $3.35-3.40$ & $0.0370-0.0453$ & 3.38 & 0.0412 \\
\hline
\end{tabular}

Table 5. Lift and drag coefficients computed with the $k-\zeta$ model for the Lockheed CC airfoil tested by Novak $\left(C_{\mu}=0.10,\left(C_{L}\right)_{\exp }=3.58\right)$.

\begin{tabular}{|c|c|c|c|}
\hline $\begin{array}{c}\text { Grid } \\
\text { Number }\end{array}$ & $\begin{array}{c}\text { Grid } \\
\text { Level }\end{array}$ & $C_{L}$ & $C_{D}$ \\
\hline 7 & coarse & 4.22 & 0.0787 \\
7 & medium & 4.32 & 0.0609 \\
\hline
\end{tabular}

Table 6. Lift and drag coefficients computed time accurately with the SST model for the Lockheed CC airfoil tested by Novak $\left(C_{\mu}=0.10,\left(C_{L}\right)_{\exp }=3.58\right)$.

than SST, and differences probably occur because of the somewhat earlier jet separation. Like with the SST model, there are larger differences between the computed and measured shear stress profiles, as seen in Figs. 17a-17c, than those obtained with the SARC model. These differences are also most likely due to the difference between the computed and experimental separation locations.

\section{Time-Dependent Effects}

Now we return to the earlier discussion of Fig. 11, showing an unexpected and substantial change in the pressure distribution computed on grid 7 using the SST model. This sudden change in the solution after several grid refinements is a result of a premature separation of the jet from the Coanda surface. Over 20,000 multigrid cycles were performed for this calculation to ensure that there would not be a further changes in the separation location. Since there are significant oscillations in the $C_{D}$, time-accurate computations were also done on the coarse and medium levels of grid 7. As displayed in Fig. 18, the solution character on grid 7 changed when the flow was computed time-accurately: separation no longer occurred prematurely. Now, calculated surface pressure variations are similar to the ones on grids $4-6$, but the $C_{L}$ is somewhat higher (see Table 6). The higher $C_{L}$ is due to a slightly later separation that occurs in the time-accurate solution, which is indicated by the larger lower surface separation bubble seen in the streamline plot of Fig. 19b compared to Fig. 19a. In Figs. 20a-20c and Figs. 21a-21c the velocity and Reynolds stress profiles from the time-accurate solutions on grid 7 are compared with the data. In addition, results for the steady-state calculation on grid 5 using the SST model are also included, so as to easily allow delineation of the differences due to iterative procedure. The differences are generally small, but they are somewhat larger at the $\theta=110^{\circ}$ and $\theta=120^{\circ}$ locations. This is not surprising since the separation is not the same in the two types of computations. Earlier we showed the SST model's higher sensitivity to grid than other turbulence models for steady-state computations. This time-accurate computation on grid 7 demonstrates how solving time-accurately can improve the SST model's results.

Another important numerical issue regarding the time-iterative process is solution uniqueness. During the course of performing steady-state computations with various initial conditions (IC's), ranging from freestream conditions to previous solutions, we discovered that the same solution was not always obtained. For example, if the IC's are nonphysical (i.e., jet wraparound), then the solution can somtimes, although not always, remain nonphysical. Here, jet wraparound is defined as a general characterization of the flow, where the the jet stream traverses the entire Coanda surface and proceeds upstream along the airfoil lower surface. It should be pointed out that even using a turbulence model that might otherwise yield a physically reasonable solution given better IC's can also admit a nonphysical solution.

We performed some numerical experiments to investigate how the turbulence models considered in this 
paper behave when the initial solution is nonphysical. Starting with the nonphysical solution obtained on grid 2 with the SST model (see Fig. 10), we continued the computation with the SARC model. In this case the SARC model converged, and allowed recovery from the nonphysical IC's and a physically reasonable solution was obtained. Next, again starting with the nonphysical solution, we computed a solution with the $k-\zeta$ model. The solution did not converge (only reached quasi-steady state), and exhibited jet wraparound. If we instead initialized with free-stream conditions, the $k-\zeta$ solution again reached a quasi-steady state, but this time it did not contain jet wraparound. From these experiments, we see that quasi-steady solutions resulting from certain steady-state computations can be sensitive to initial conditions. This sensitivity suggests that such quasi-steady results may not necessarily be representative of a true (converged) solution.

\section{GACC Airfoil}

Next, the computational method was applied to one of the CC airfoil flow cases from the Jones et al. ${ }^{6}$ experiment. As indicated previously the Mach number is 0.10 , and the Reynolds number is $0.533 \times 10^{6}$. The jet momentum coefficient for the case being considered is about 0.093 .

In the current work there are two principal objectives in simulating the flow over the GACC airfoil. The first one is to determine if a simulation that includes the compressible effects of the jet flow also exhibits vortex shedding from the blunt lip of the jet slot. The second objective is to investigate the effects on jet detachment if this vortex shedding does occur. It is with these goals in mind that we consider the work of Baker $^{4}$ in which the incompressible RANS equations were solved for several GACC airfoil cases, and vortex shedding from the jet slot lip was observed.

An overset grid strategy was used by Baker to discretize the domain. The set of grids used included nested Cartesian grids in the far field and an O-type grid around the airfoil. The three-dimensional RANS code (called CFDSHIP-IOWA) applied by Baker required five spanwise planes for two-dimensional simulations. Each spanwise plane consisted of 76,372 grid points for steady-state computations. Free-stream conditions were prescribed at the outer boundary, and symmetry was applied in the spanwise direction. Results were obtained for $C_{\mu}=0.013,0.021,0.041,0.059,0.091$, and 0.162 . For time-accurate calculations a 2-D wind tunnel simulation was done, still using five spanwise planes. In each spanwise plane there were 46,347 grid points. Solutions were computed with the lower and upper boundaries in a spanwise plane being inviscid walls, where a nonpenetration (tangency) boundary condition was enforced. Symmetry conditions were again used in the spanwise direction. Note that while this 2-D wind tunnel approach could account partially for the blockage effects, it cannot account for the tunnel sidewall effects in the experiment. Thus, it does not eliminate the need for angle of attack corrections. There was grid clustering at the airfoil leading edge as well as at the jet slot and its lip. Simulations were done with second-order spatial and temporal approximations. Upwind differencing was used for the advection terms. Thus, for both the Baker and the present computations there is comparable numerical accuracy.

As in the computations of Baker, the present steady-state calculations using the SST turbulence model produced potentially unsteady solutions, as indicated by the oscillatory behavior in the aerodynamic coefficient histories shown in Figs. 22 and 23. However, in Ref. 4 (at essentially the same $C_{\mu}$ ) the jet moves from the Coanda surface onto the lower surface of the airfoil. We do not observe such wraparound behavior using the SST model, as seen in Figs. 24 and 25, which are snapshots of the streamlines for the medium and fine grid steady-state results. However, this wraparound behavior was encountered when we applied the SARC model (see Fig. 26). Using the fine level of grid 1 with this model does not eliminate the jet wraparound. On the other hand, if no rotation/curvature correction is applied (i.e., reducing to SA model), there is wraparound on the medium level of Grid 1 but not on the fine level, as shown in Figs. 27 and 28.

Some steady-state computations were also done on grid 2 for the GACC airfoil. Grid 2 has nearly twice as many grid points as Grid 1 on the Coanda surface in the streamwise direction. Once again there are significant oscillations in the low magnitude drag coefficients. Table 7 presents the computed $C_{L}$ and $C_{D}$ values. The $C_{L}$ on the fine level of grid 2 is approximately $12 \%$ lower than the $C_{L}$ on the fine level of grid 1. This result indicates that further refinement is needed in the streamwise direction to attain grid independent aerodynamic coefficients.

Time-accurate calculations were also performed for the $C_{\mu}=0.093$ case. For these computations on medium and fine grids, a very small nondimensional time step was used: $\Delta t$ of 0.001 , where $\Delta t$ is scaled by the ratio of chord to free-stream speed of sound. With this $\Delta t$ a free-stream disturbance requires 10,000 time steps to traverse the length of the airfoil. This small $\Delta t$ was used in order to capture possible high-frequency vortex shedding from the lip of the jet slot. The lift and drag coefficients converge in these calculations, as 


\begin{tabular}{|c|r|c|c|r|c|}
\hline $\begin{array}{c}\text { Grid } \\
\text { Number }\end{array}$ & $\begin{array}{c}\text { Grid } \\
\text { Level }\end{array}$ & $\begin{array}{c}\text { Range of } \\
C_{L}\end{array}$ & $\begin{array}{c}\text { Range of } \\
C_{D}\end{array}$ & $\begin{array}{c}\text { Average } \\
C_{L}\end{array}$ & $\begin{array}{c}\text { Average } \\
C_{D}\end{array}$ \\
\hline 1 & medium & $2.97-3.00$ & $0.0210-0.0518$ & 2.98 & 0.0364 \\
1 & fine & $3.45-3.51$ & $0.0270-0.0974$ & 3.48 & 0.0622 \\
2 & medium & $3.15-3.27$ & $0.0547-0.0770$ & 3.21 & 0.0609 \\
2 & fine & $3.01-3.02$ & $0.0377-0.0474$ & 3.02 & 0.0426 \\
\hline
\end{tabular}

Table 7. Lift and drag coefficients computed with the SST model for the GACC airfoil tested by Jones $\left(C_{\mu}=0.093\right)$.

\begin{tabular}{|c|c|c|c|}
\hline $\begin{array}{c}\text { Grid } \\
\text { Number }\end{array}$ & $\begin{array}{c}\text { Grid } \\
\text { Level }\end{array}$ & $C_{L}$ & $C_{D}$ \\
\hline 1 & medium & 2.90 & 0.0296 \\
1 & fine & 3.44 & 0.0766 \\
\hline
\end{tabular}

Table 8. Lift and drag coefficients computed time accurately with the SST model for the GACC airfoil tested by Jones $\left(C_{\mu}=0.093\right)$.

evident in Figs. 29 and 30. In Figs. 31 and 32 the streamlines for the medium and fine grids are displayed. On the medium grid there is no separation on the airfoil lower surface, whereas for the fine grid solution there is significant flow separation occurring at approximately $x / c=0.8-0.9$. This seems to occur because the fine grid jet is separating later; this characteristic behavior is similar to what was seen earlier for the Lockheed case. For both calculations two isolated vortices appear behind the blunt lip of the jet slot. These vortices are shown for the fine grid in Fig. 33, and there is no vortex shedding. In the incompressible results of Baker, solutions for all $C_{\mu} \geq 0.021$ considered exhibit shedding of the vortices at estimated frequencies between $67 \mathrm{kHz}$ and $128 \mathrm{kHz}$. In Fig. 34 the Mach contours for the fine grid calculation are displayed. Clearly, the flow is compressible in the neighborhood of the jet slot.

In Fig. 35 the medium and fine grid pressure distributions are compared with experimental data. Table 8 presents the corresponding lift and drag coefficients. The fine grid results show a larger leading edge suction peak than the data. Moreover, it indicates suction levels higher than the data over the entire upper surface of the airfoil except at the trailing edge, where the suction peak is underpredicted. This result may indicate that the predicted separation location of the jet is too far around the Coanda surface. The overprediction of the suction peak is not surprising since there was no angle of attack correction applied to the geometric angle of attack of zero degrees. However, a modest angle of attack correction would primarily affect the suction level in the vicinity of the leading edge suction peak. There is also the conflicting problem that the computed trailing edge suction peak is lower than the experimental one. This suggests that the $C_{\mu}$ for the computation is not high enough, but increasing $C_{\mu}$ would enhance circulation and make the suction levels on the airfoil upper surface even higher. Certainly, if the computed flow were to separate sooner, then this conflict could be resolved.

In Baker, ${ }^{4}$ the jet for the circular trailing edge GACC airfoil was modeled for most of the cases via specification of a boundary condition at the jet exit location. Here, we explore the use of the same boundary condition to compare with results that included the modeled cavity. The equation for the velocity curve fit was:

$$
\begin{aligned}
& U=V_{\text {jet }} \cos \Theta U_{\text {poly }} \\
& V=V_{\text {jet }} \sin \Theta U_{\text {poly }}
\end{aligned}
$$

where $V_{\text {jet }}=0.718$ for the particular case investigated, $\Theta=-18^{\circ}$, and 


$$
\begin{aligned}
U_{\text {poly }} & =-1.2222 \times 10^{2} \hat{y}^{10}-1.7043 \times 10^{2} \hat{y}^{9}+1.8036 \times 10^{3} \hat{y}^{8}-3.4603 \times 10^{3} \hat{y}^{7} \\
& +2.9482 \times 10^{3} \hat{y}^{6}-1.0602 \times 10^{3} \hat{y}^{5}-9.7236 \times 10^{1} \hat{y}^{4}+2.2944 \times 10^{2} \hat{y}^{3} \\
& -8.5386 \times 10^{1} \hat{y}^{2}+1.4472 \times 10^{1} \hat{y}+0.0036
\end{aligned}
$$

where $\hat{y}$ is the normalized distance across the slot. Figure 36 shows a comparison of the total velocity profile specified at the jet exit using the 10th degree polynomial compared with a profile from CFD results that modeled the cavity. The two agree closely.

When using the 10th degree polynomial boundary condition in a steady-state computation with the SST turbulence model, results did not converge and the unconverged flowfield near the slot exit was very chaotic. By computing time-accurately, the flowfield near the slot exit "smoothed out" and became regular, and the jet wrapped smoothly around the curved trailing edge all the way to near the lower lip. This is in contrast to the results of Baker, for which time-accurate computations yielded a distinct vortex shedding behavior near the slot exit when the 10th-degree polynomial boundary condition was employed.

Streamlines for the SST result using the 10th-degree polynomial boundary condition are displayed in Fig. 37. In comparing these streamlines with those when the plenum is used (see Fig. 31) we see that the jet moves farther around the Coanda surface when the plenum is not modeled.

\section{Vortex Shedding}

Even though the GACC airfoil has a blunt jet slot lip, it does not necessarily follow that vortex shedding will occur. There are specific criteria that determine the existence of self-excited vortex shedding. In the following discussion we first delineate what these requirements are; and then, we examine the present results to see if vortex shedding is indicated.

In Khorrami et al. ${ }^{21}$ the problem of vortex shedding from a blunt slat trailing edge of a multielement airfoil is addressed. A discussion of the criteria for vortex shedding based on theoretical investigations is given. Here, we provide the key points of that discussion. From theoretical analysis of flow past a flat plate with a blunt trailing edge, $\mathrm{Koch}^{22}$ postulated that a resonance-like mechanism controls the self-excited vortex shedding process, and this mechanism is associated with a region of local absolute instability. Koch also found that wake symmetry plays an important role with respect to wake stability. Moreover, for asymmetrical wakes, there appears to be a limiting asymmetry, beyond which there is no absolute instability but only convective instability. The analysis of Koch has been confirmed by the direct numerical simulation of the same flat plate flow by Hammond and Redekopp. ${ }^{23}$ As pointed out by Khorrami et al., this simulation has established the direct link between vortex shedding and the characteristics of the wake global instability.

Korrami et al. considered two cases, one with a $30^{\circ}$ slat deflection and the other with a $20^{\circ}$ deflection. For the first case there was vortex shedding from the blunt slat trailing edge, and in the other case no shedding occurred. It is important to distinguish between these two cases, since this will facilitate the evaluation of the behavior in the present results. In each case a vertical cut of streamwise velocity in the wake was made. For the $30^{\circ}$ slat deflection case there was a strong reverse flow on the centerline of the near wake. This reverse flow vanishes in 2-3 trailing-edge thicknesses downstream of the trailing edge. Also, the wake was nearly symmetrical with the bottom stream being slightly stronger. With the $20^{\circ}$ deflection reverse flow was still detected, but its magnitude and downstream distance were much smaller. The bottom stream dominated in this particular case, leading to a highly asymmetric wake.

For the present time-accurate GACC airfoil computations, the velocity of the jet (bottom stream) is about three times that of the flow leaving the upper surface of the airfoil (top stream), resulting in a significantly asymmetrical wake. Let $H$ be the jet slot lip thickness (approximately equal to the jet slot height). On the wake centerline the reverse flow magnitude (scaled by the free-stream speed of sound) is about -0.025 at one $H$ downstream of the lip. This reverse flow disappears in 2-3 $H$ downstream of the lip. Since the criteria for vortex shedding only provide qualitative guidelines rather than quantitative requirements, the assessment given here can only suggest that self-excited vortex shedding may not occur. It should be mentioned that the first point off the surface of the blunt lip is $1.0 \times 10^{-5}$ chords. This grid spacing is half that used by Baker. ${ }^{4}$ 


\section{Concluding Remarks}

Several numerical issues pertaining to the computation of CC airfoil flows have been considered. The numerical issues have been separated in two principal areas. One area is mesh refinement effects, and the other area is time-dependent or iterative procedure effects. Three turbulence models have been used in investigating the issues in these areas. These models include the one-equation Spalart-Allmaras (SA) model with curvature correction (SARC), the two-equation shear-stress transport (SST) model of Menter, and the two-equation $k-\zeta$ model, where $\zeta$ represents enstrophy.

A sequence of meshes has been used in computing flow over a Lockheed CC airfoil for the particular case in which the jet momentum coefficient $C_{\mu}=0.10$. Solutions have been compared with the detailed experimental data of Novak. Results obtained with the SARC model and the $k-\zeta$ model have both exhibited consistency with mesh refinement. On the other hand, the SST model has exhibited inconsistent behavior. In particular, we have shown that this model is very sensitive to streamwise grid resolution around the Coanda surface. We have demonstrated that the SST model's high sensitivity to grid can make it difficult to draw conclusions regarding its solution behavior when performing steady-state computations. Our steady-state computation on the finest grid produced results that were very different in character from the solutions on other reasonably fine grids. This jump in behavior due to grid refinement did not appear to be related to improved resolution of any particular aspect of the flow. It highlighted the importance of using time-iterative solutions (rather than steady-state) for this type of flowfield for the SST model on fine grids.

Two effects on the Lockheed CC airfoil calculations due to the time-iterative process have been examined. One effect is that a time-accurate computation can produce a reasonable solution (relative to experimental data) when a steady-state computation does not. We have demonstrated such behavior when the SST model is used. Another effect concerns nonunique solutions. Numerical experiments have been performed that verify that very different quasi-steady solutions can occur, particularly when initial conditions have the jet wrapping around (unphysically) to the airfoil lower surface. These experiments suggest that quasi-steady results may not necessarily be representative of the true (converged) solution.

Both steady-state and time-accurate computations have been done for the GACC airfoil. The present time-accurate solutions for the compressible RANS equations do not exhibit self-excited vortex shedding from the jet slot lip. Theoretical criteria only suggest that shedding may not occur. Also, the current timeaccurate solutions do not show any dramatic change in the jet detachment location seen with the steady-state calculations. The present results are in contrast to those of Baker, who solved the incompressible rather than the compressible RANS equations.

A number of researchers have encountered nonphysical solutions when computing $\mathrm{CC}$ airfoil flows with various turbulence models. In these solutions a jet wraparound occurs, where the jet traverses the entire Coanda (circular trailing edge) surface and at least part of the airfoil lower surface. We have shown for two different CC airfoil flows that insufficient mesh resolution is at least partially responsible for this solution behavior. However, simple grid refinement does not always cure this problem. Additional work is reqired to determine a complete remedy for such nonphysical behavior.

The results of this paper suggest that the use of RANS computations for predicting CC flows on Coanda surfaces is far from being straightforward. As demonstrated both by the results presented here, as well as by results from earlier workshops (e.g., CC workshop ${ }^{1}$ held at NASA Langley in 2004) and other studies, ${ }^{3,19,20}$ traditional turbulence models sometimes work and sometimes do not, and computational solutions tend to be highly sensitive to numerical parameters and methods of solution. 


\section{References}

${ }^{1}$ Jones, G. S., and Joslin, R. D (editors), Proceedings of Circulation Control Workshop, NASA CP 2005-213509, Hampton, Virginia, March 2004.

${ }^{2}$ Abramson, J., "Two-Dimensional Subsonic Wind Tunnel Evaluation of Two Related Cambered 15-Percent Circulation Control Airfoils," DTNSRDC ASED-373, 1977.

${ }^{3}$ Swanson, R. C., Rumsey, C. L. and Anders, S. G. "Progress Towards Computational Method for Circulation Control Airfoils," AIAA Paper 2005-0089, Jan. 2005.

${ }^{4}$ Baker, W., "Simulations of Coanda Effect Circulation Control Using Reynolds-Averaged Navier-Stokes Computational Fluid Dynamics," M. S. Thesis, The Pennsylvania State University, 2004.

${ }^{5}$ Novak, C. J., Cornelius, K. C, Roads, R. K., "Experimental Investigations of the Circular Wall Jet on a Circulation Control Airfoil," AIAA Paper 87-0155, 1987.

${ }^{6}$ Jones, G. S., Viken, S. A, Washburn, A. E., Jenkins, L. N., Cagle, C. M., "An Active Flow Circulation Controlled Flap Concept for General Aviation Aircraft Applications," AIAA Paper 02-3157, 2002.

${ }^{7}$ Krist, S. L., Biedron R. T. and Rumsey, C. L., "CFL3D User's Manual," NASA TM 1998-208444, 1998.

${ }^{8}$ Roe, P., "Approximate Riemann Solvers, Parameter Vectors, and Difference Schemes," J. Comp. Phys., vol. 43, 1981, pp. 357-372.

${ }^{9}$ Spalart, P. R. and Allmaras, S. R., "A One-Equation Turbulence Model for Aerodynamic Flows," La Recherche Aerospatiale, vol. 1, 1994, pp. 5-21.

${ }^{10}$ Spalart, P. R. and Shur, M., "On the Sensitization of Turbulence Models to Rotation and Curvature," Aerospace Sci. Technol., vol. 5, 1997, pp. 297-302.

${ }^{11}$ Rumsey, C. L., Gatski, T. B., Anderson, W. K. and Nielsen, E. J., "Isolating Curvature Effects in Computing WallBounded Turbulent Flows," Inter. J. of Heat and Fluid Flow, vol. 22, 2001, pp. 573-582.

${ }^{12}$ Menter, F. R., "Improved Two-Equation $k-\omega$ Turbulence Model for Aerodynamic Flows," NASA TM $103975,1992$.

${ }^{13}$ Menter, F. R., "Zonal Two Equation $k-\omega$ Turbulence Model for Aerodynamic Flows," AIAA Paper 93-2906, 1993.

${ }^{14}$ Menter, F. R., "Two-Equation Eddy-Viscosity Turbulence Models for Engineering Applications," AIAA J. vol. 32, No. 8, 1994, pp. 1598-1605.

${ }^{15}$ Robinson, D. F., and Hassan, H. A., "Further Development of the $k-\zeta$ (Enstrophy) Turbulence Closure Model," $A I A A$ J., vol. 36, No. 10, 1998, pp. 1825-1833.

${ }^{16}$ Swanson, R. C., Rumsey, C. L, Anders, S. G., "Aspects of Numerical Simulation of Circulation Control Airfoils," Proceedings of Circulation Control Workshop, NASA CP 2005-213509, Hampton, Virginia, March 2004.

${ }^{17}$ Rumsey, C. L., Gatski, T. B. and Morrison, J. H., "Turbulence Model Predictions of Strongly Curved Flow in a U-Duct," AIAA J., vol. 38, No. 8, 2000, pp. 1394-1402.

${ }^{18}$ Abramson, J., and Rogers, E., "High-Speed Characteristics of Circulation Control Airfoils," AIAA Paper 83-0265, 1983.

${ }^{19}$ Slomski, J. F., Gorski, J. J, Miller, R. W., Marino, T. A., "Numerical Simulation of Circulation Control Airfoils as Affected by Turbulence Models," AIAA Paper 2002-0851, Jan. 2002.

${ }^{20}$ Paterson, E. G, and Baker, W. J., "Simulation of Steady Circulation Control for Marine-Vehicle Control Surfaces," AIAA Paper 2004-0748, Jan. 2004.

${ }^{21}$ Khorrami, M. R., Berkman, M. E. and Choudhari, C., "Unsteady Flow Computations of a Slat with a Blunt Trailing Edge," AIAA J., vol. 38, No. 11, 2000, pp. 2050-2058.

${ }^{22}$ Koch, W. "Local Instability Characteristics and Frequency Determination of Self-Excited Wake Flows," J. of Sound and Vibration, vol. 99, No. 1, 1985, pp. 53-83.

${ }^{23}$ Hammond, D. A., and Redekopp, L. G.. "Global Dynamics of Symmetric and Asymmetric Wakes," J. of Fluid Mech., vol. 331, 1997, pp. 231-260. 


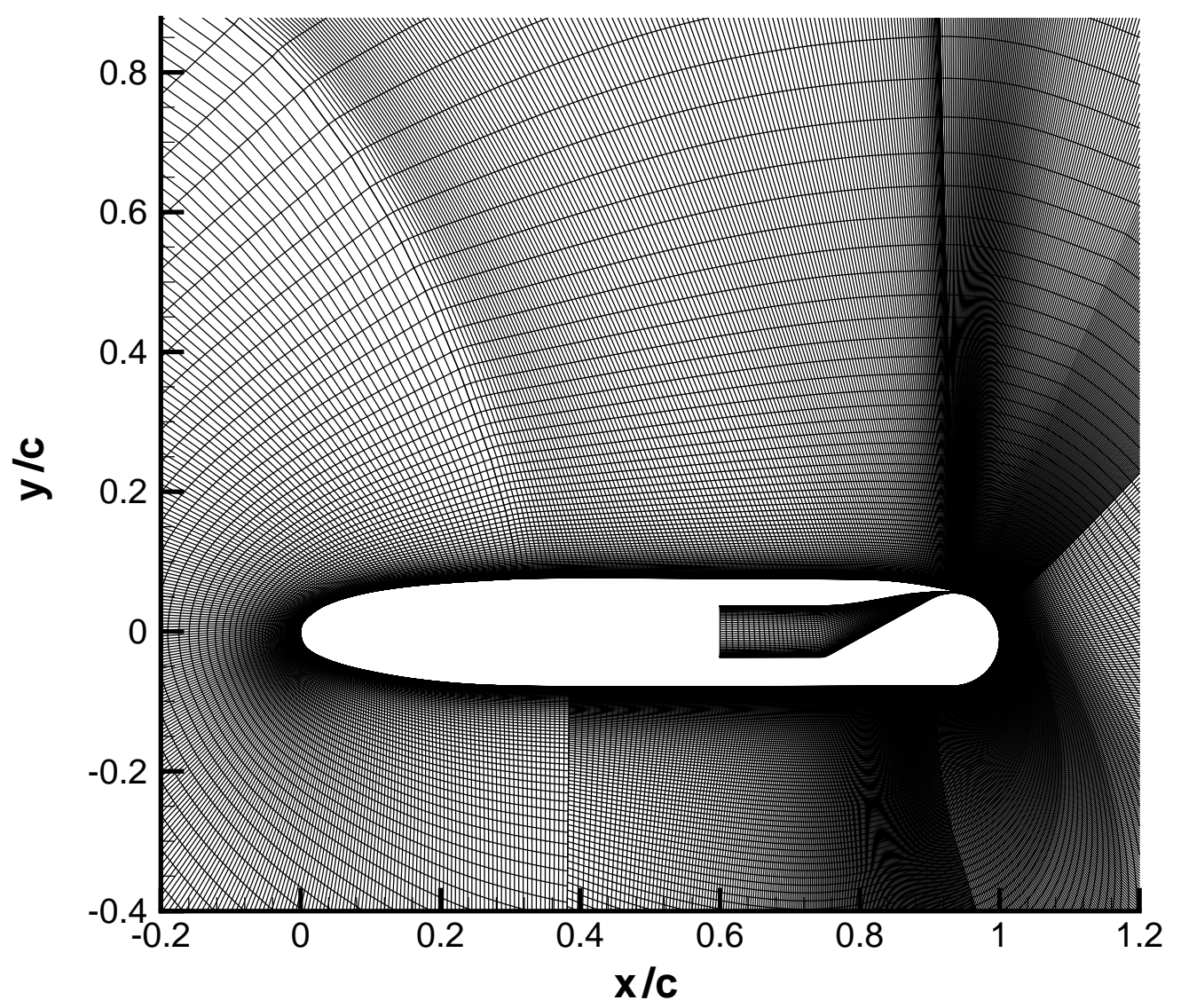

Figure 1. Partial view of Lockheed grid 4 (fine). 


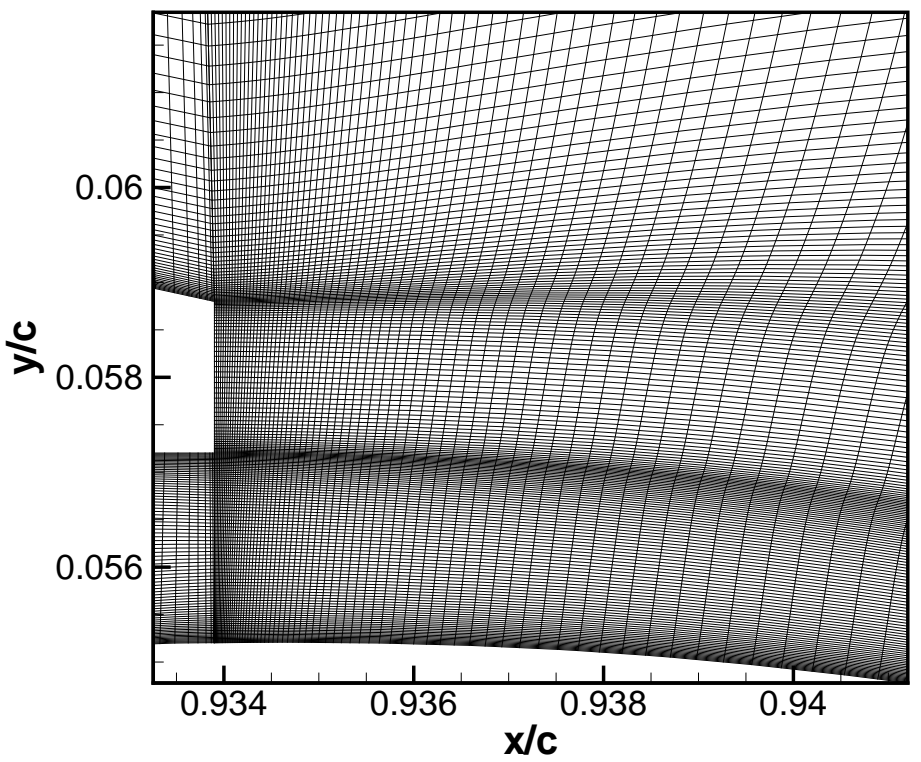

Figure 2. Lockheed grid 4 (fine) in the vicinity of the lip of the jet slot.

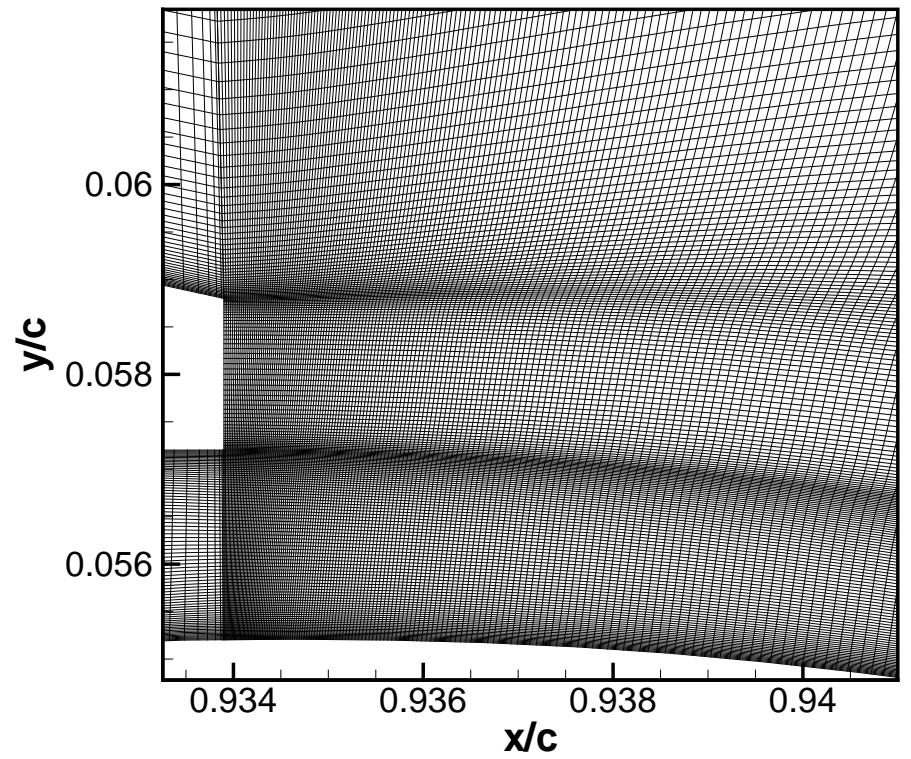

Figure 3. Lockheed grid 5 (fine) in the vicinity of the lip of the jet slot. 


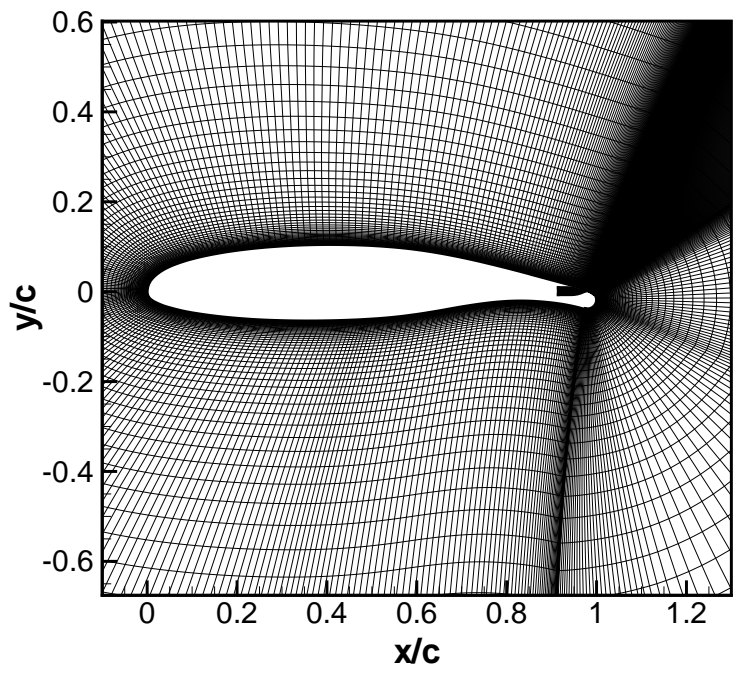

Figure 4. Partial view of grid 1 (fine) for GACC airfoil.

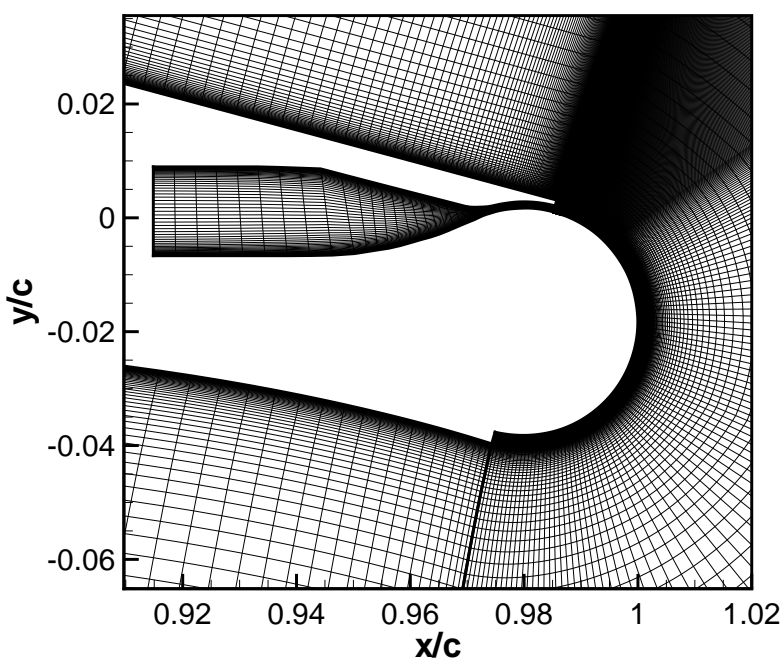

Figure 5. Grid 1 (fine) in vicinity of trailing edge of GACC airfoil. 


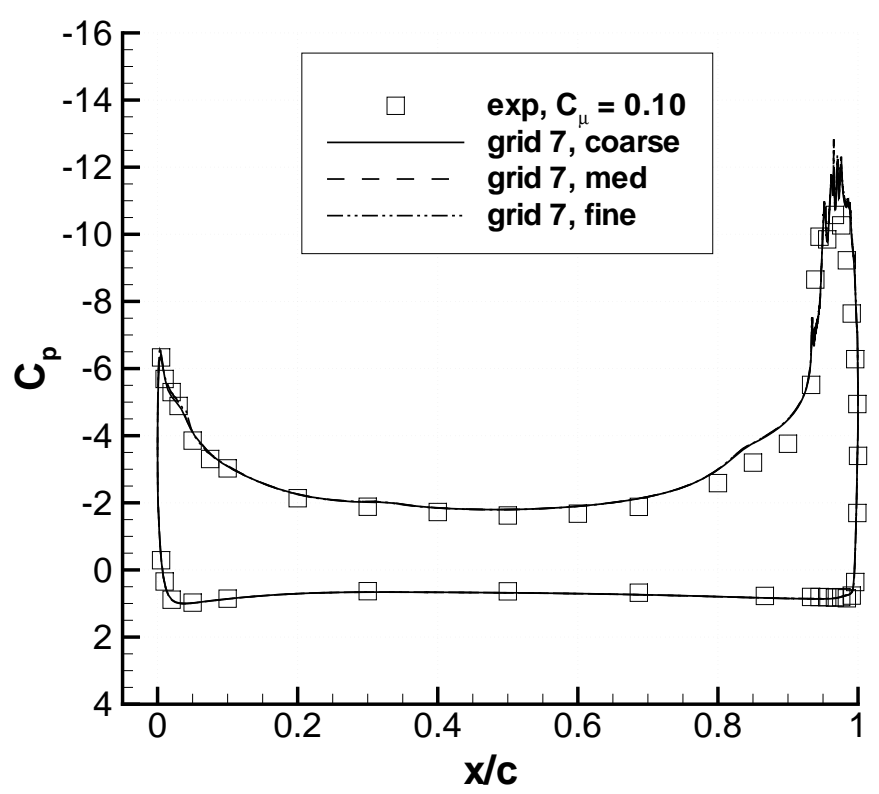

Figure 6. Lockheed case surface pressures computed with SARC model $\left(M_{\infty}=0.12, \alpha=-5.86^{\circ}, R e_{c}=0.986 \times 10^{6}\right.$, $\left.C_{\mu}=0.10\right)$.

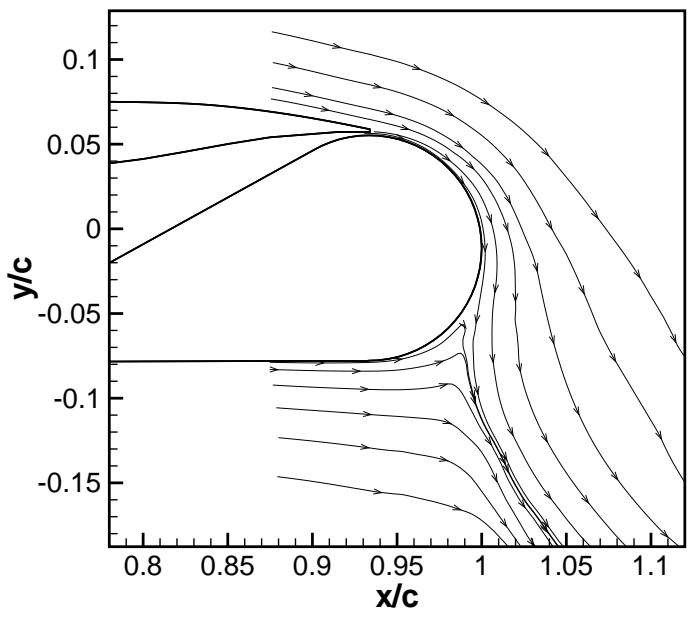

(a)

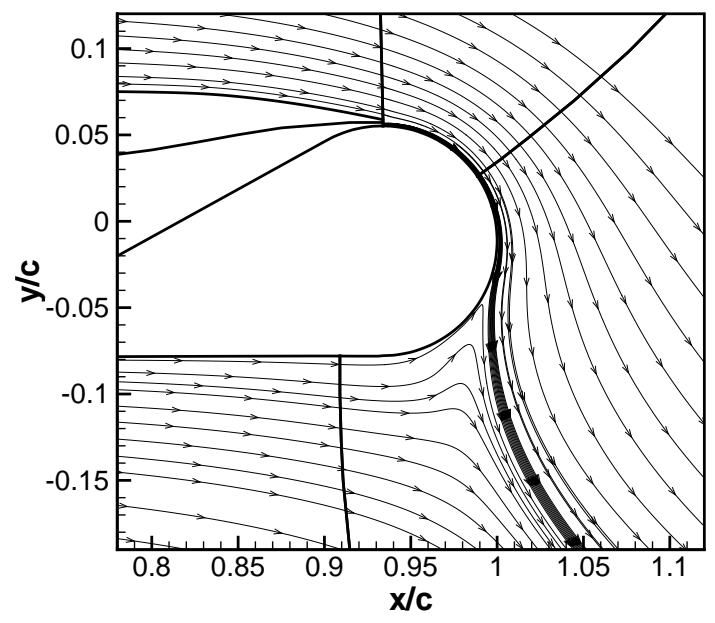

(b)

Figure 7. Lockheed case streamlines, $C_{\mu}=0.10$ : (a) experiment, (b) computation, SARC model, grid 7: fine (solid lines indicate zone boundaries in the computation). 

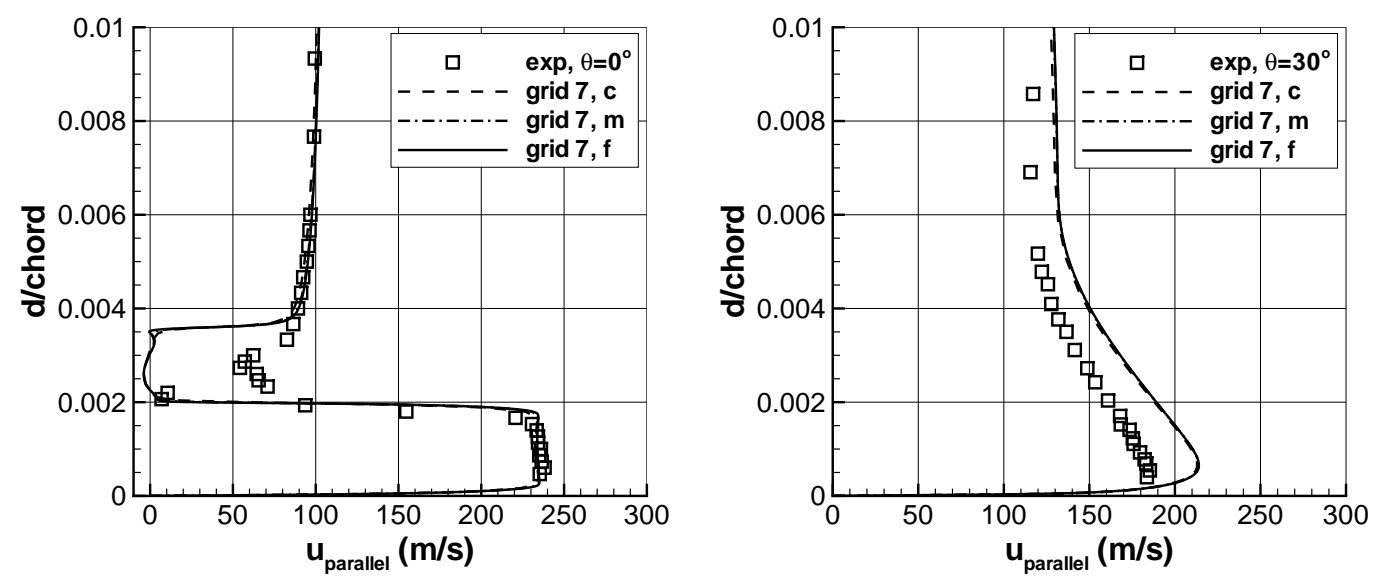

Figure 8a. Lockheed case velocity profiles, $C_{\mu}=0.10$, SARC model, $\theta=0,30$ degrees.
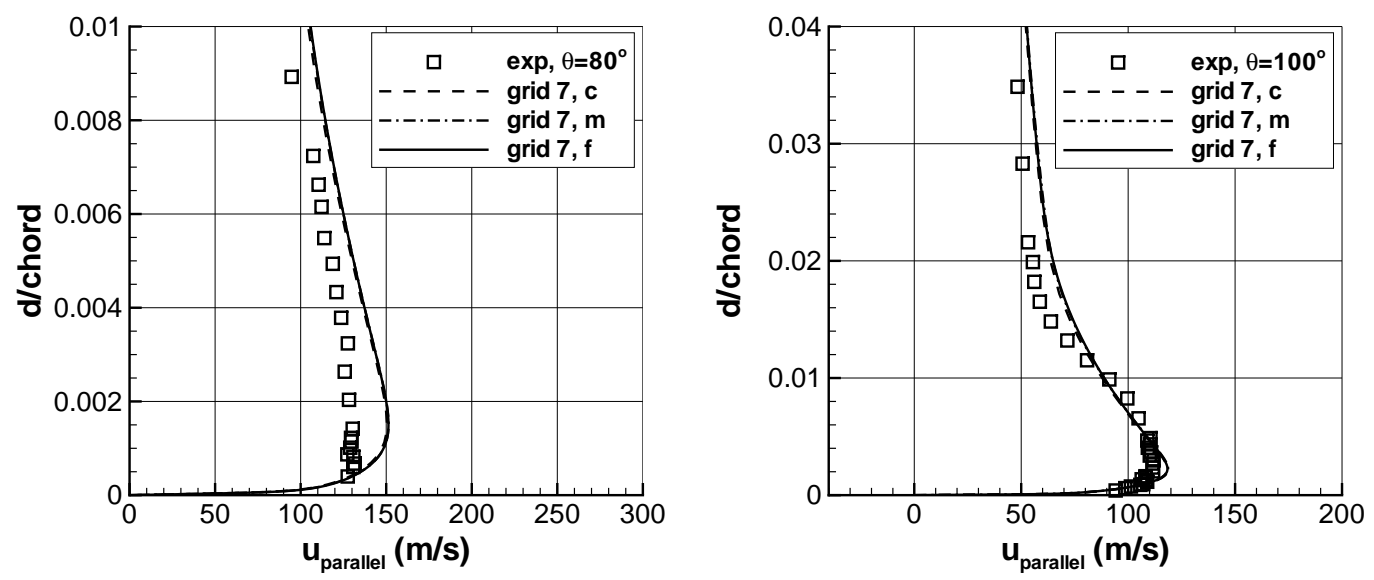

Figure 8b. Lockheed case velocity profiles, $C_{\mu}=0.10$, SARC model, $\theta=80,100$ degrees.
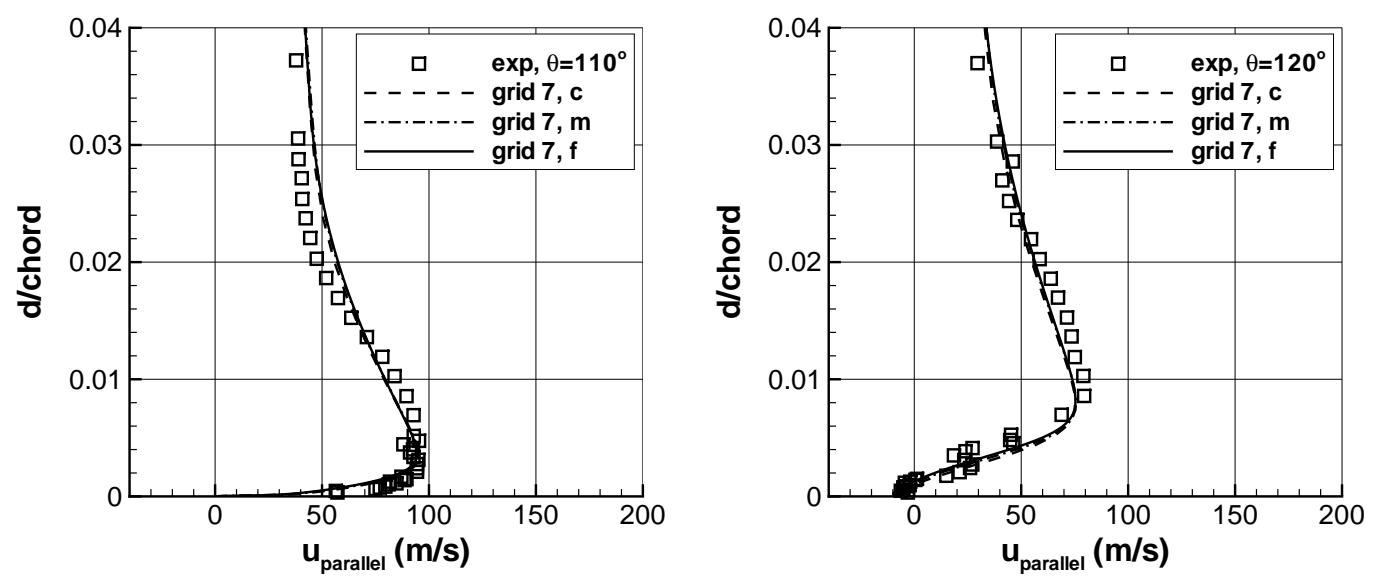

Figure 8c. Lockheed case velocity profiles, $C_{\mu}=0.10$, SARC model, $\theta=110,120$ degrees. 

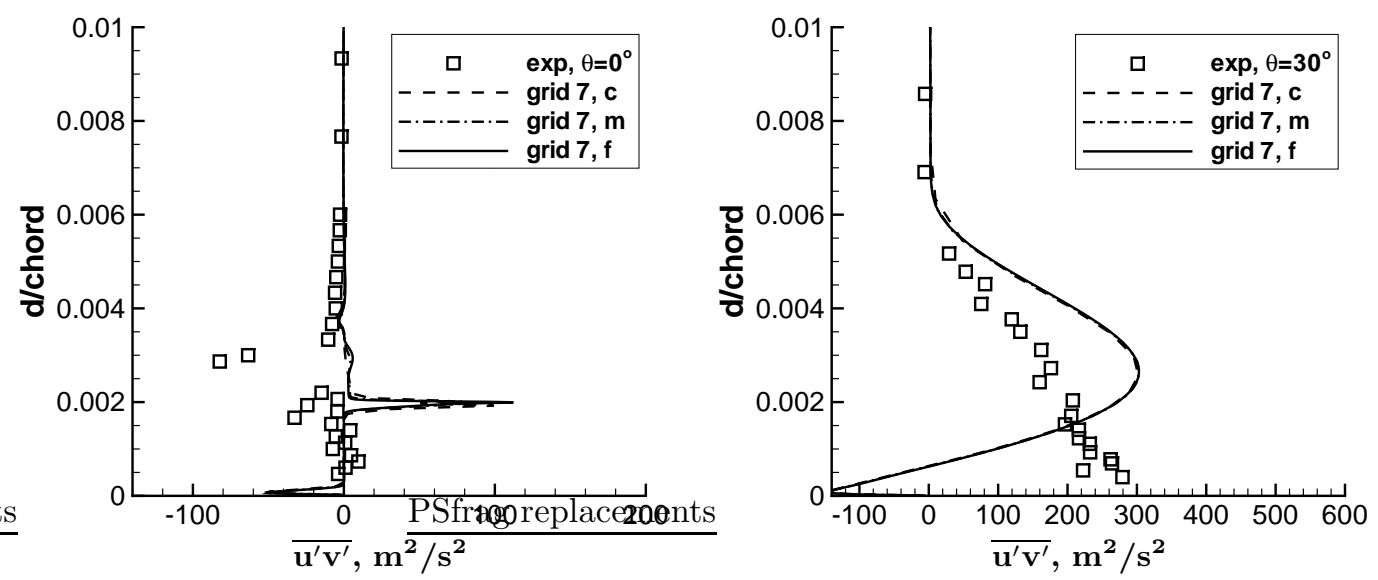

Figure 9a. Lockheed case shear stress profiles, $C_{\mu}=0.10$, SARC model, $\theta=0,30$ degrees.
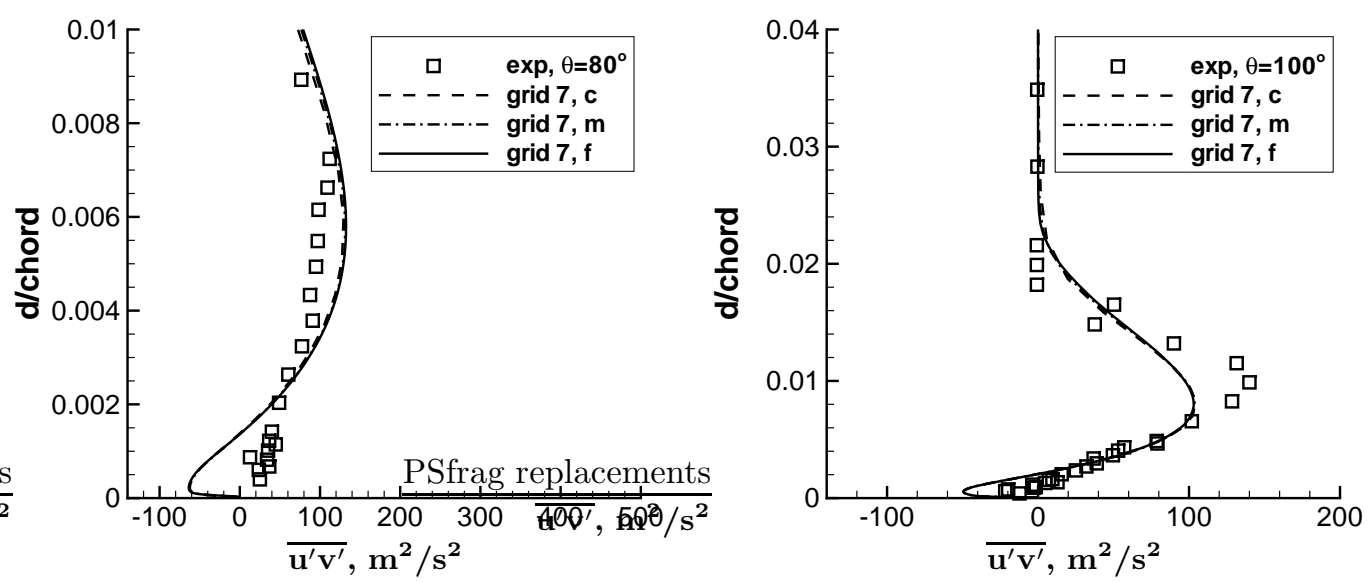

Figure 9b. Lockheed case shear stress profiles, $C_{\mu}=0.10$, SARC model, $\theta=80,100$ degrees.
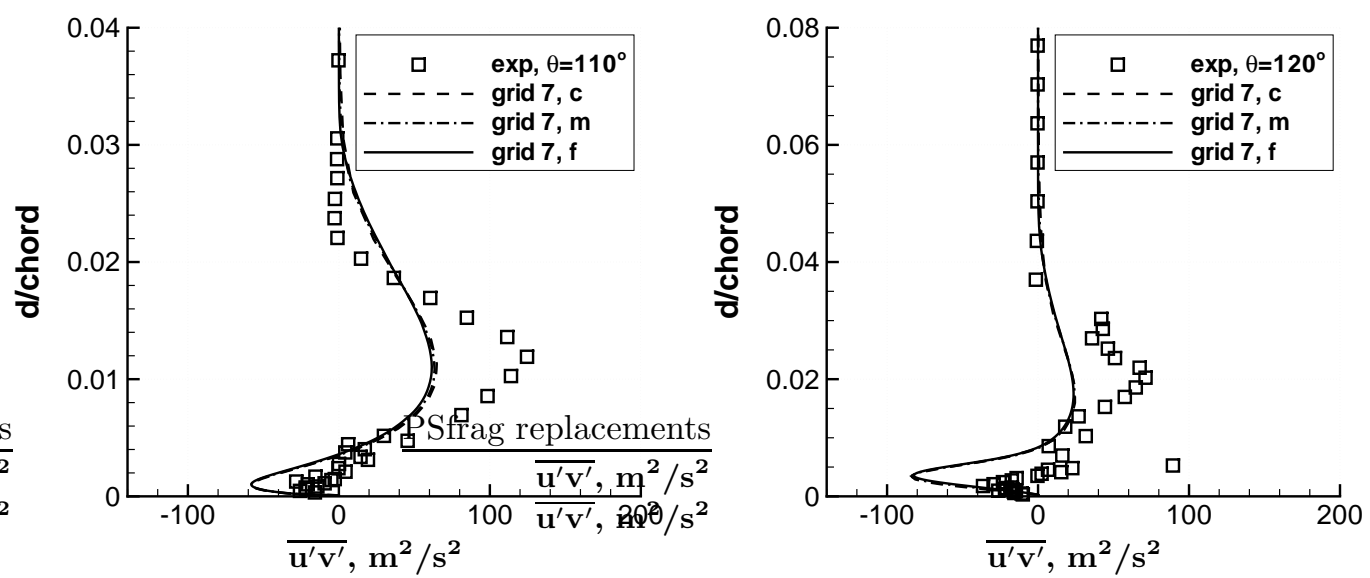

Figure 9c. Lockheed case shear stress profiles, $C_{\mu}=0.10$, SARC model, $\theta=110,120$ degrees. 


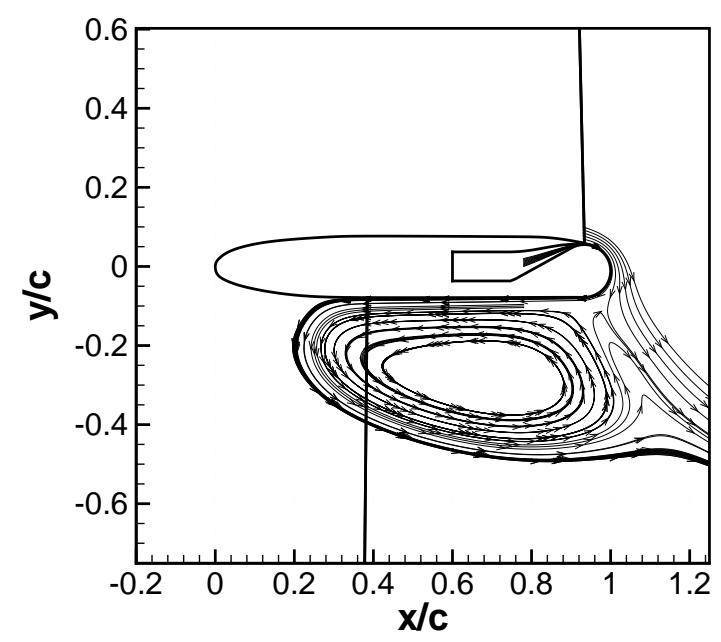

(a)

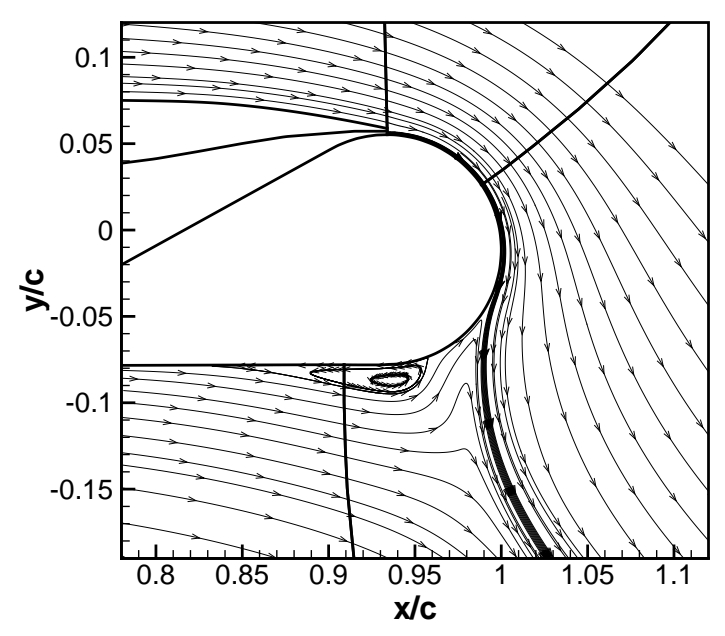

(b)

Figure 10. Lockheed case streamlines, computations with SST model, $C_{\mu}=0.10$ : (a) grid 2: medium, (b) grid 5: fine.

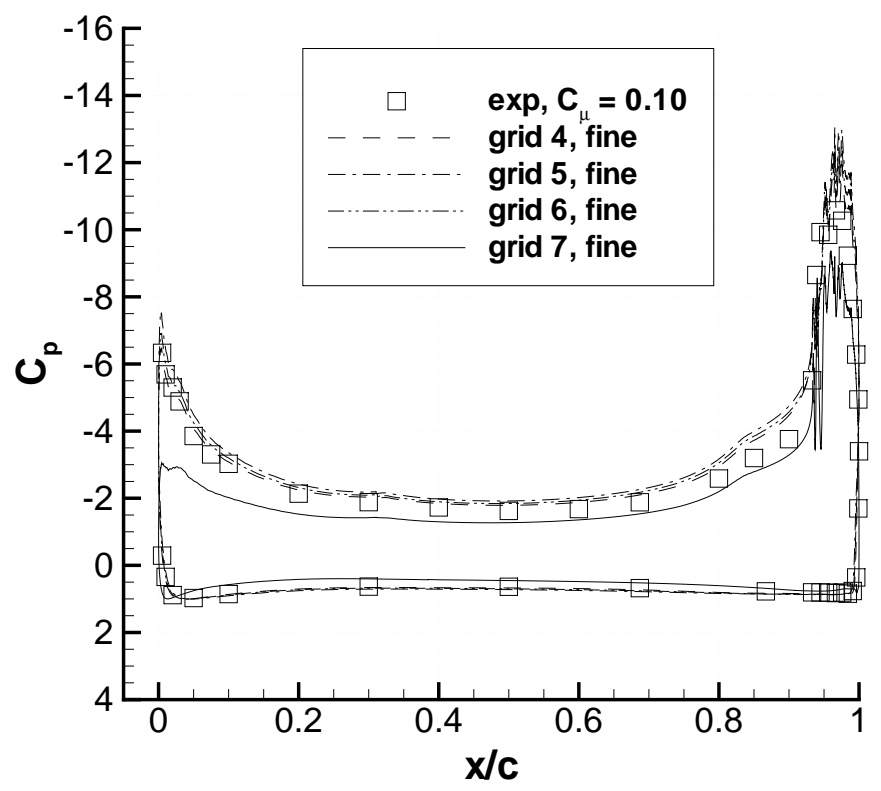

Figure 11. Lockheed case surface pressures computed with SST model $\left(M_{\infty}=0.12, \alpha=-5.86^{\circ}, R_{c}=0.986 \times 10^{6}\right.$, $\left.C_{\mu}=0.10\right)$. 

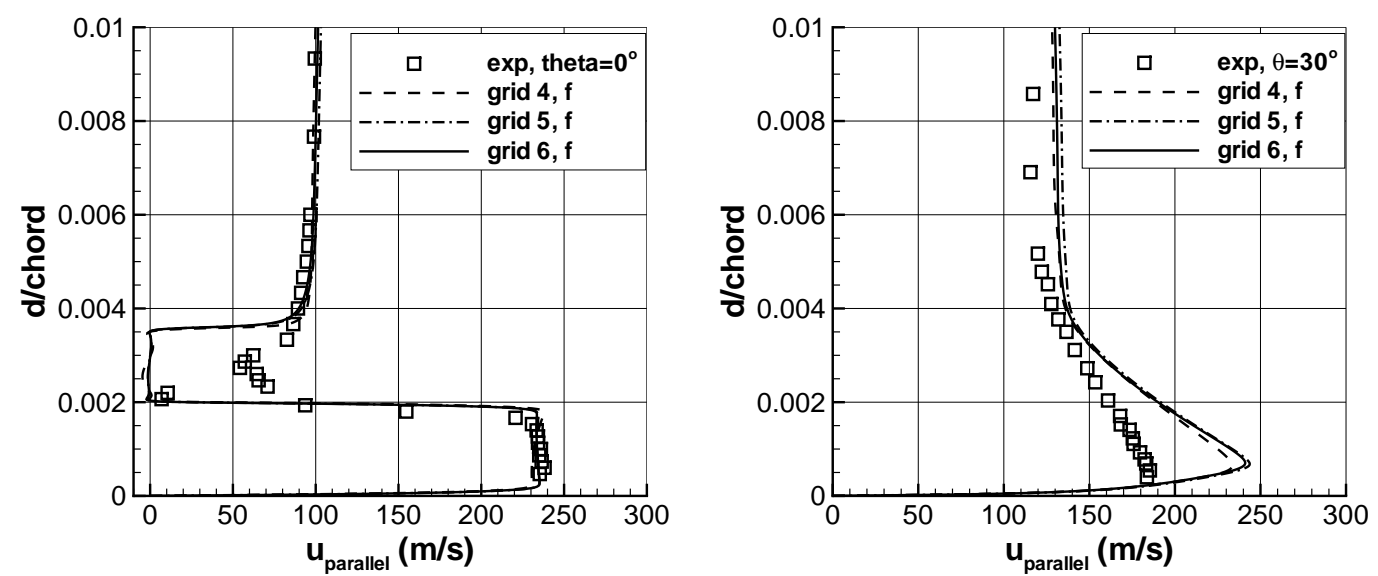

Figure 12a. Lockheed case velocity profiles, $C_{\mu}=0.10$, SST model, $\theta=0,30$ degrees.
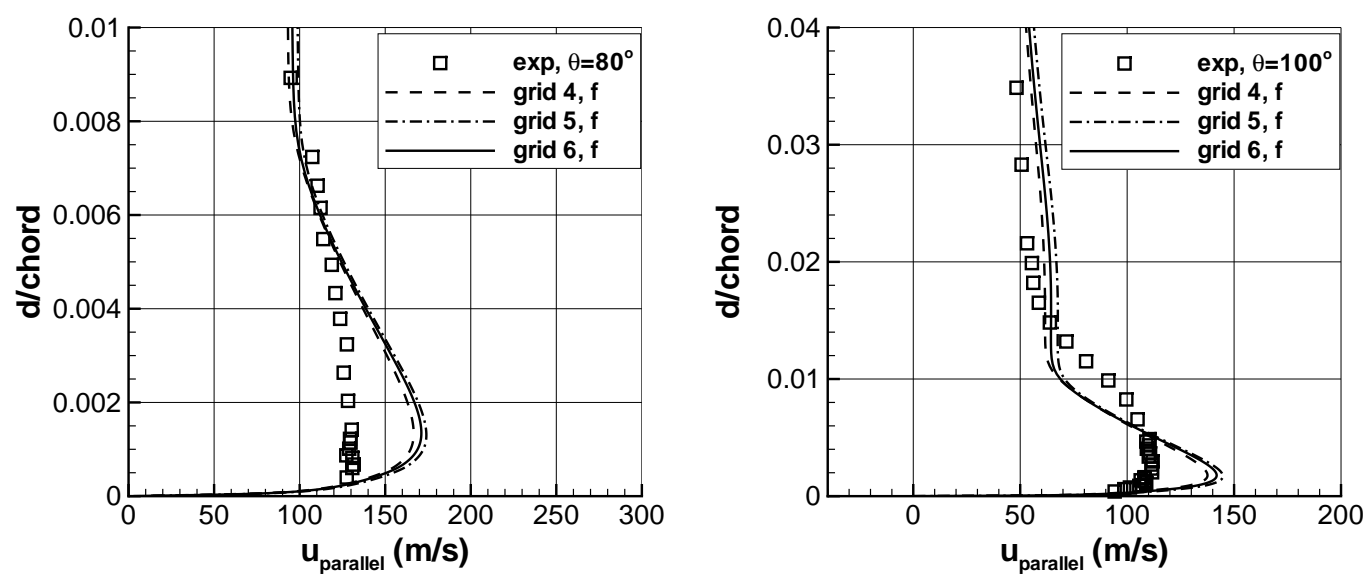

Figure 12b. Lockheed case velocity profiles, $C_{\mu}=0.10$, SST model, $\theta=80,100$ degrees.
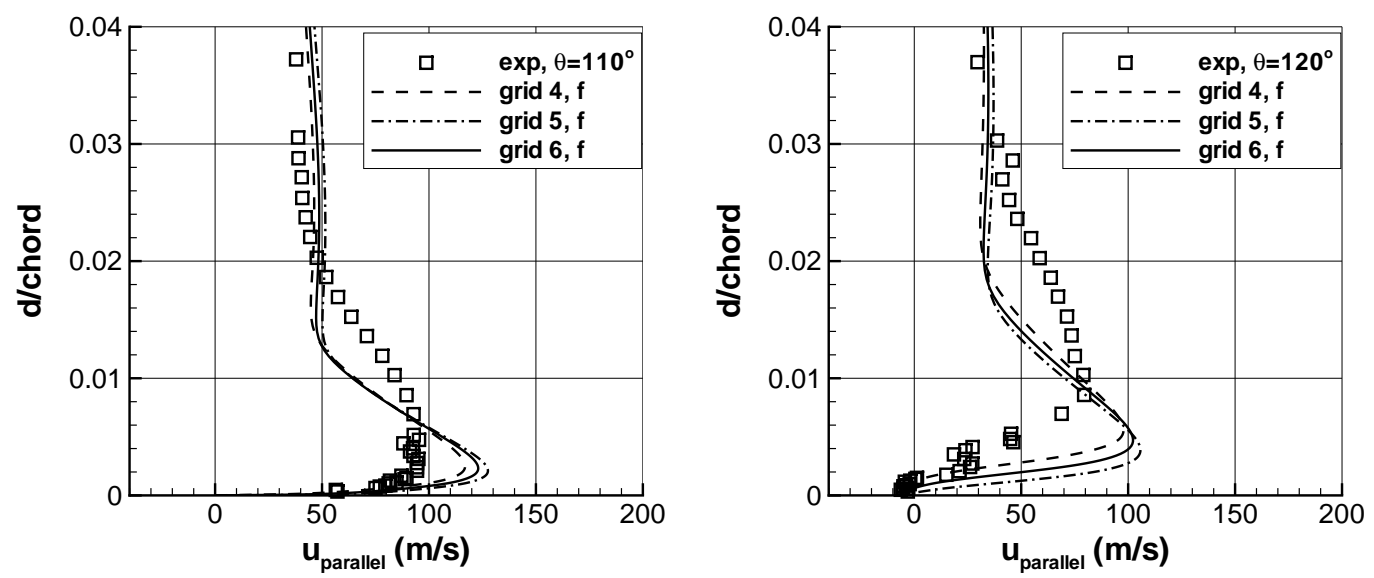

Figure 12c. Lockheed case velocity profiles, $C_{\mu}=0.10$, SST model, $\theta=110,120$ degrees. 

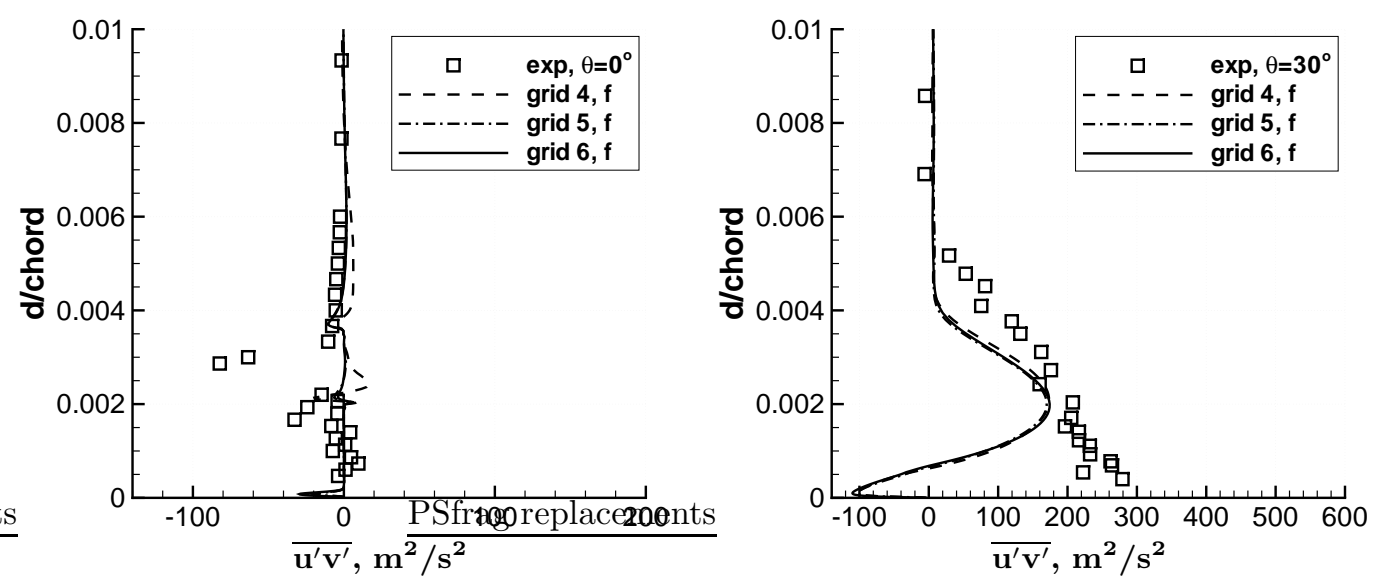

Figure 13a. Lockheed case shear stress profiles, $C_{\mu}=0.10$, SST model, $\theta=0,30$ degrees.
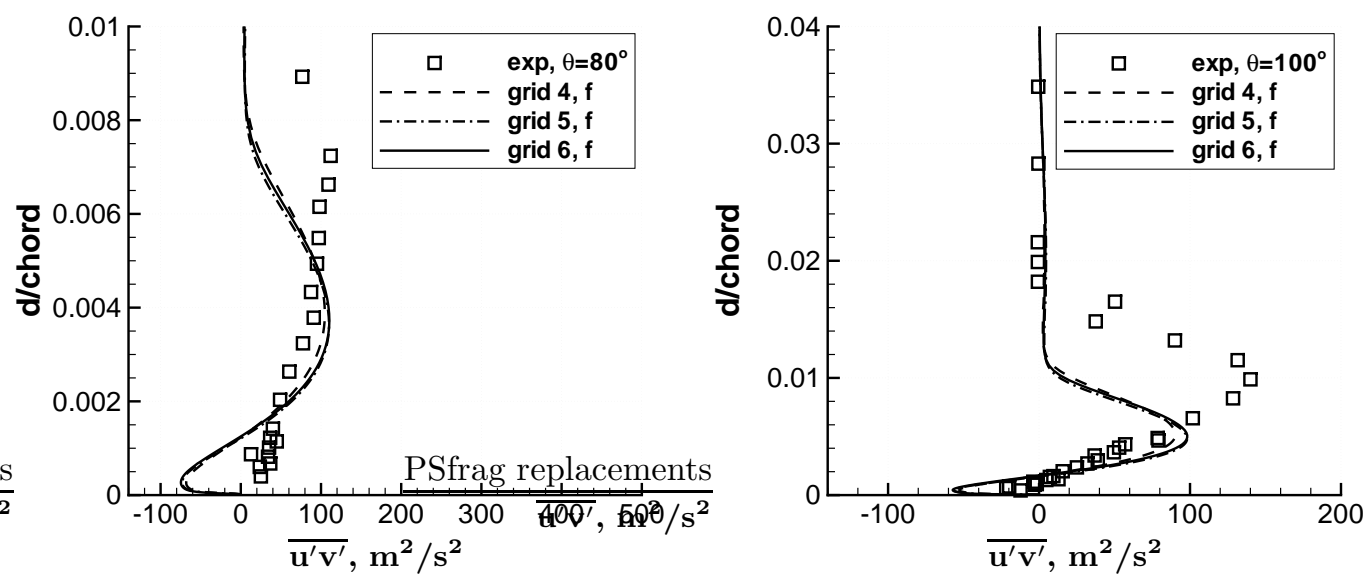

Figure 13b. Lockheed case shear stress profiles, $C_{\mu}=0.10$, SST model, $\theta=80,100$ degrees.
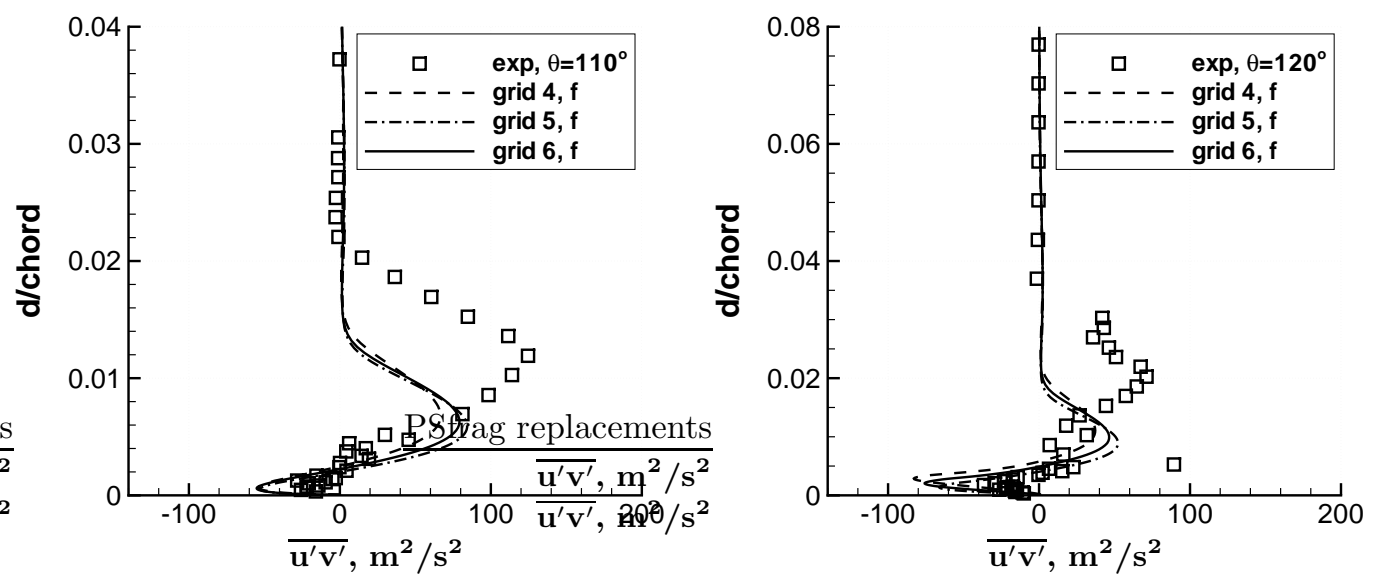

Figure 13c. Lockheed case shear stress profiles, $C_{\mu}=0.10$, SST model, $\theta=110,120$ degrees. 


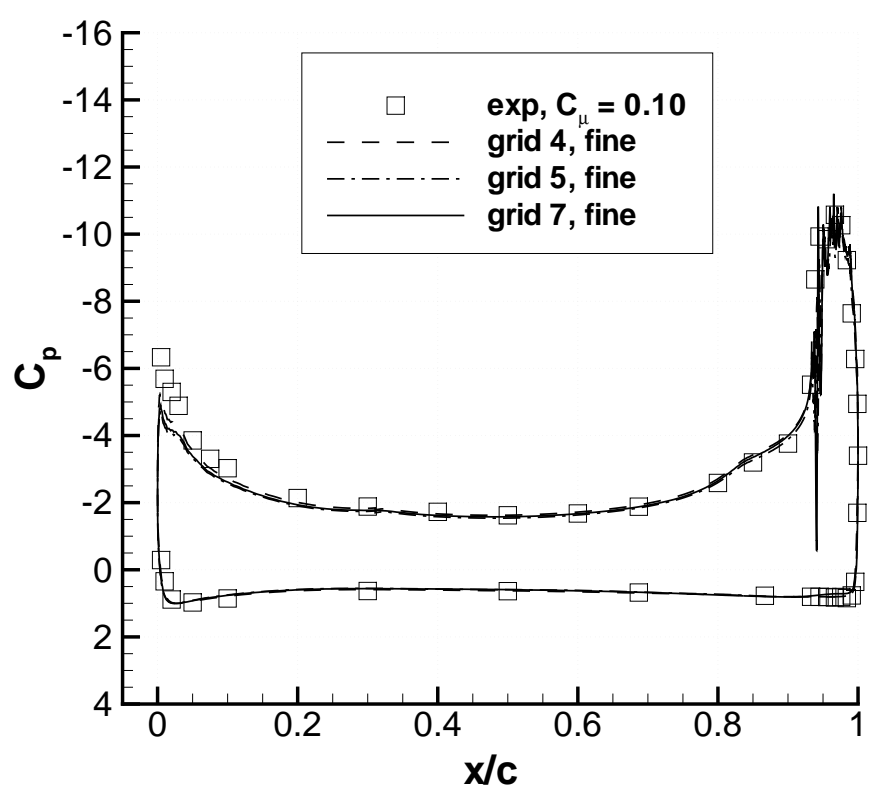

Figure 14. Lockheed case surface pressures computed with k- $\zeta$ model $\left(M_{\infty}=0.12, \alpha=-5.86^{\circ}, R e_{c}=0.986 \times 10^{6}\right.$, $\left.C_{\mu}=0.10\right)$.

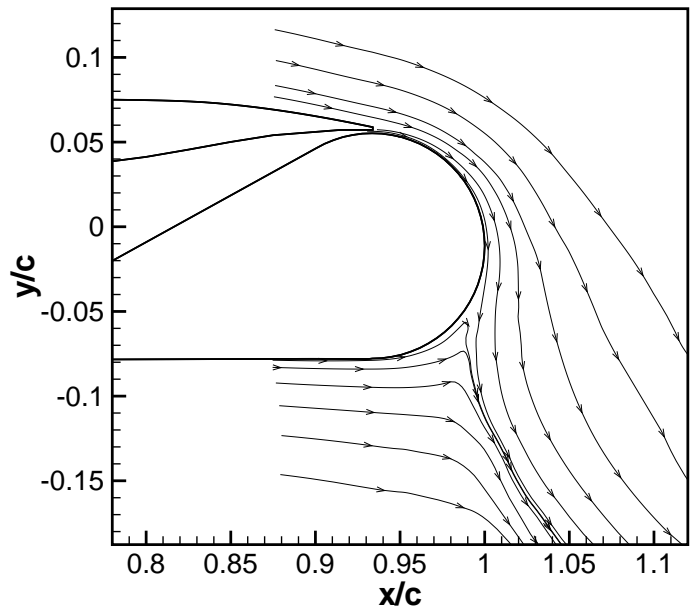

(a)

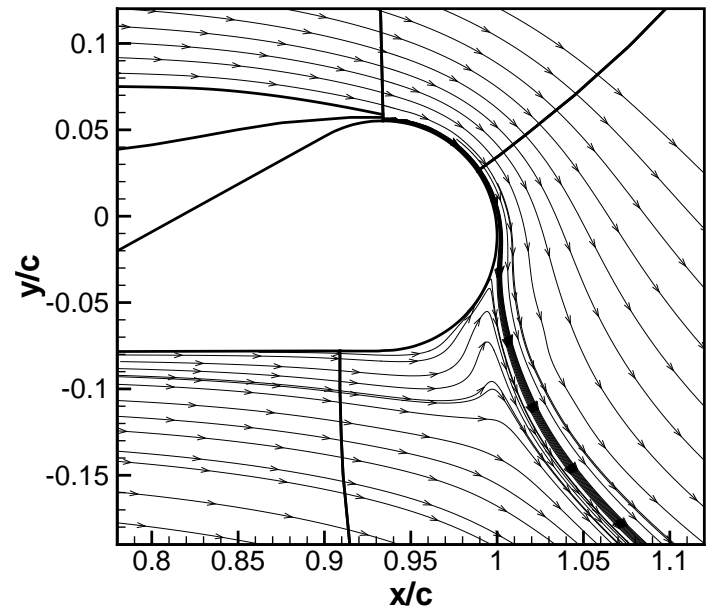

(b)

Figure 15. Lockheed case streamlines, $C_{\mu}=0.10$ : (a) experiment, (b) computation, k- $\zeta$ model, grid 7: fine. 

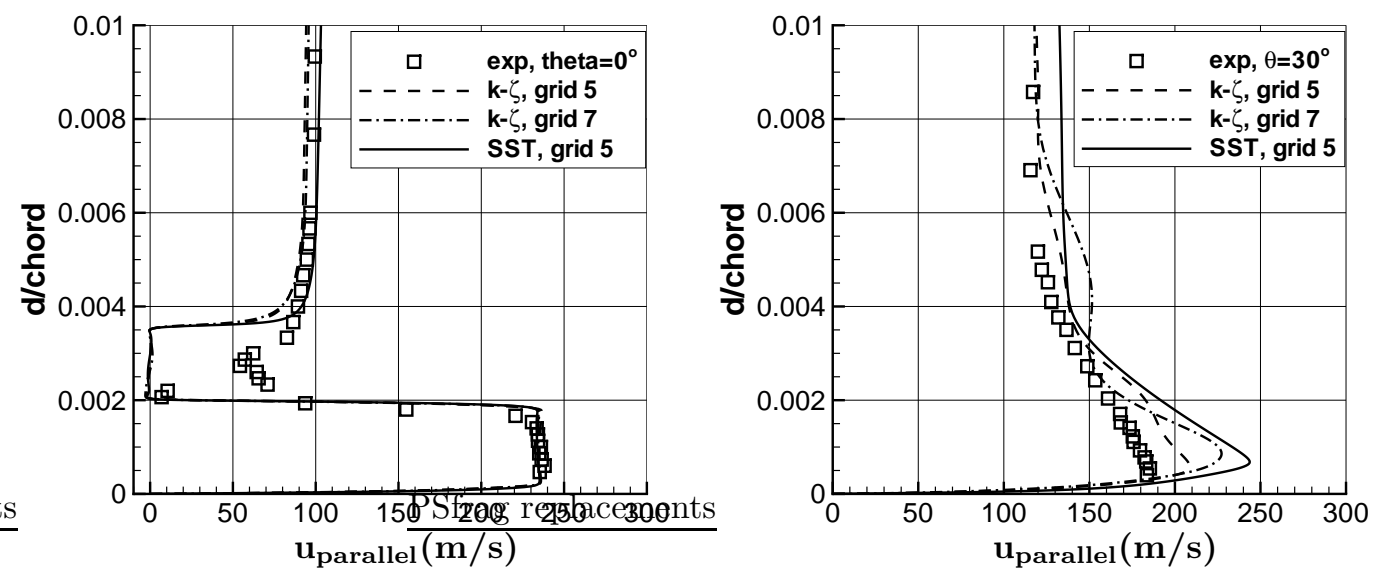

Figure 16a. Lockheed case velocity profiles, $C_{\mu}=0.10, \mathrm{k}-\zeta$ and SST models, fine grids, $\theta=0,30$ degrees.
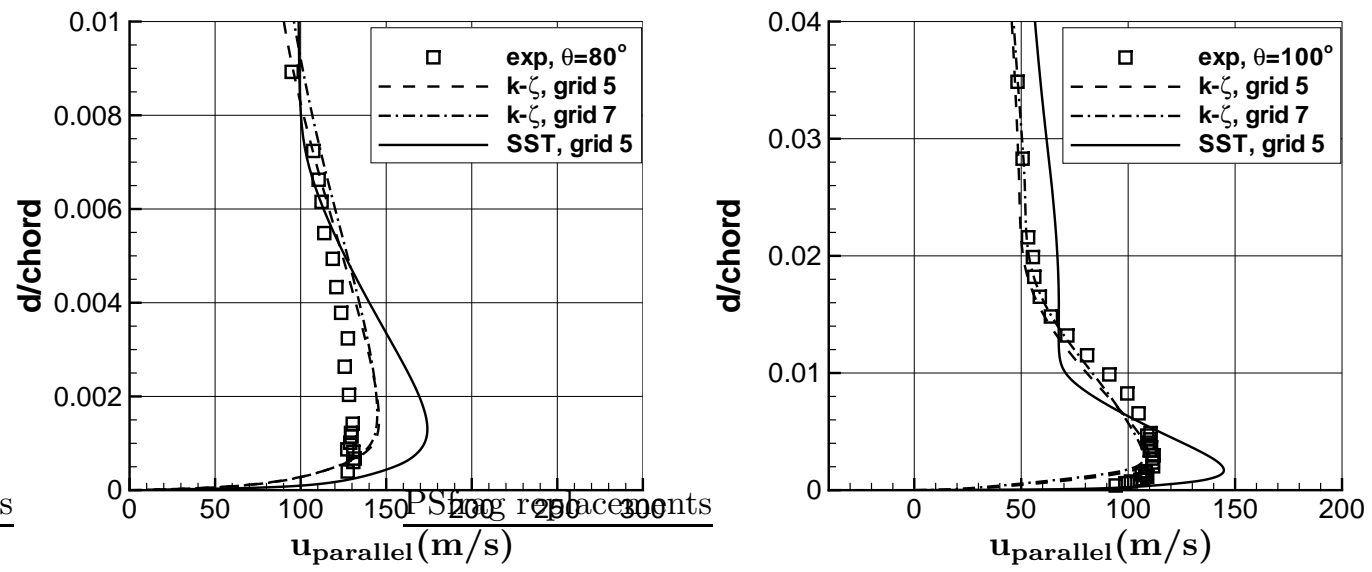

Figure 16b. Lockheed case velocity profiles, $C_{\mu}=0.10, \mathrm{k}-\zeta$ and SST models, fine grids, $\theta=80,100$ degrees.
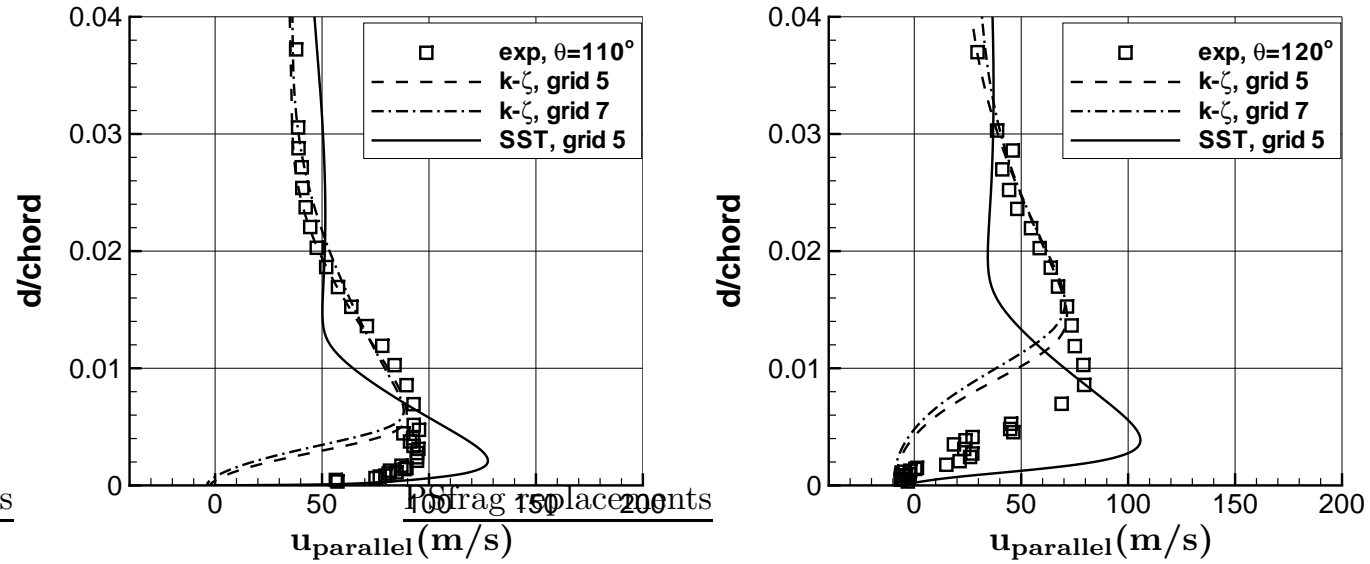

Figure 16c. Lockheed case velocity profiles, $C_{\mu}=0.10, \mathrm{k}-\zeta$ and SST models, fine grids, $\theta=110,120$ degrees. 

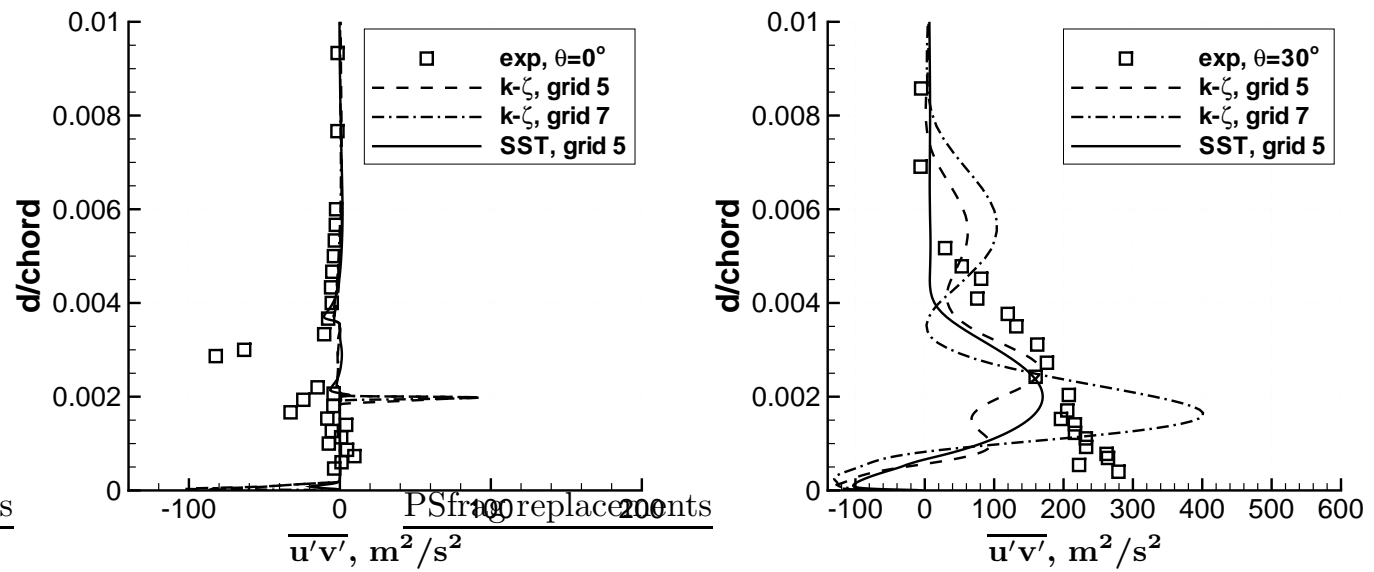

Figure 17a. Lockheed case shear stress profiles, $C_{\mu}=0.10$, k- $\zeta$ and SST models, fine grids, $\theta=0,30$ degrees.
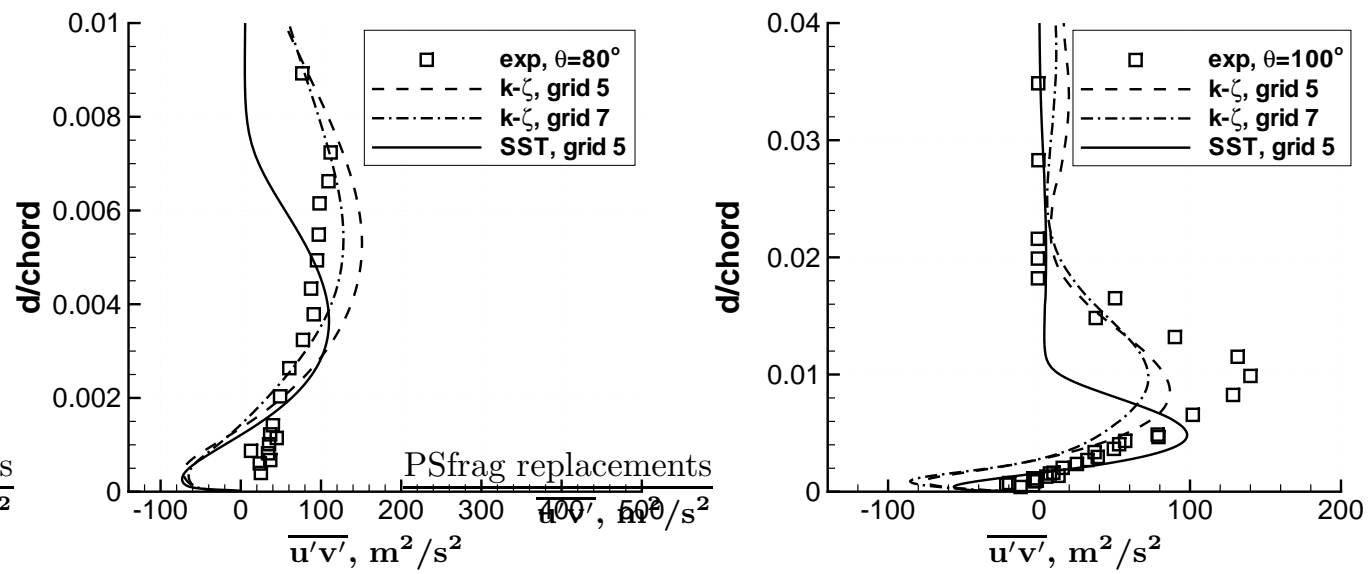

Figure 17b. Lockheed case shear stress profiles, $C_{\mu}=0.10, \mathrm{k}-\zeta$ and SST models, fine grids, $\theta=80,100$ degrees.
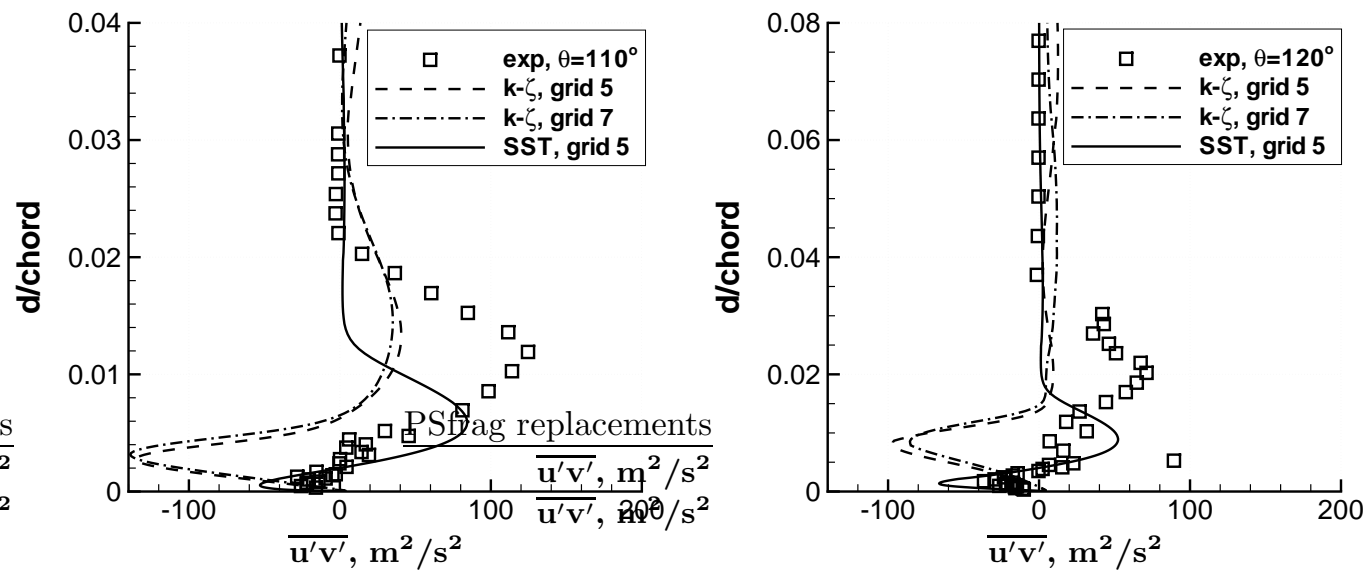

Figure 17c. Lockheed case shear stress profiles, $C_{\mu}=0.10$, k- $\zeta$ and SST models, fine grids, $\theta=110,120$ degrees. 


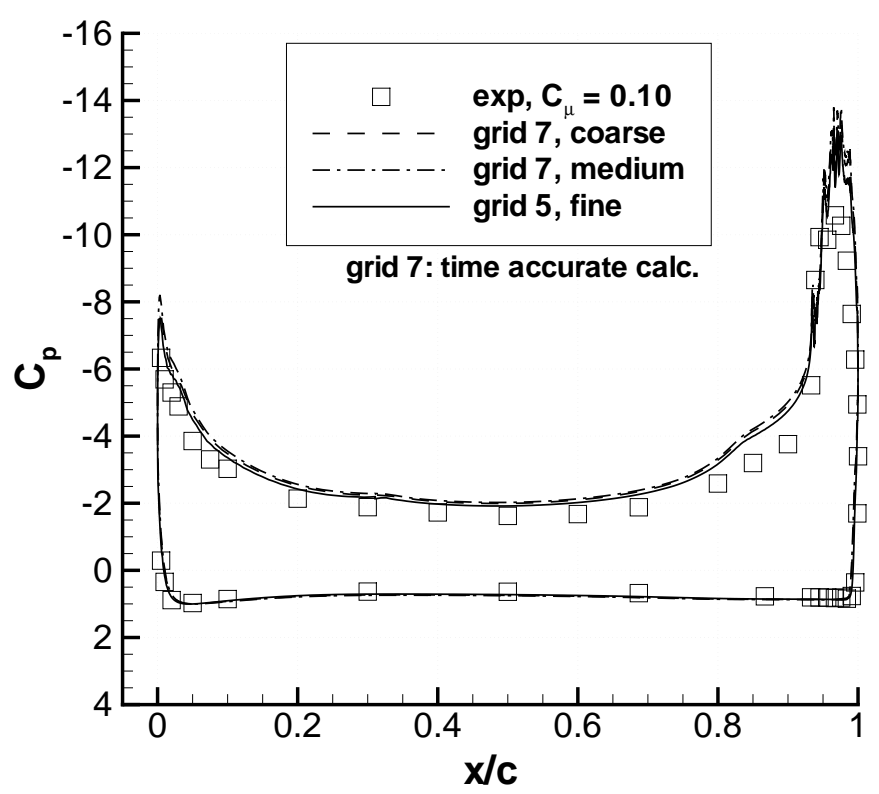

Figure 18. Lockheed case surface pressures computed with SST model; time accurate calculations on grid 7 $\left(M_{\infty}=0.12, \alpha=-5.86^{\circ}, R e_{c}=0.986 \times 10^{6}, C_{\mu}=0.10\right)$.

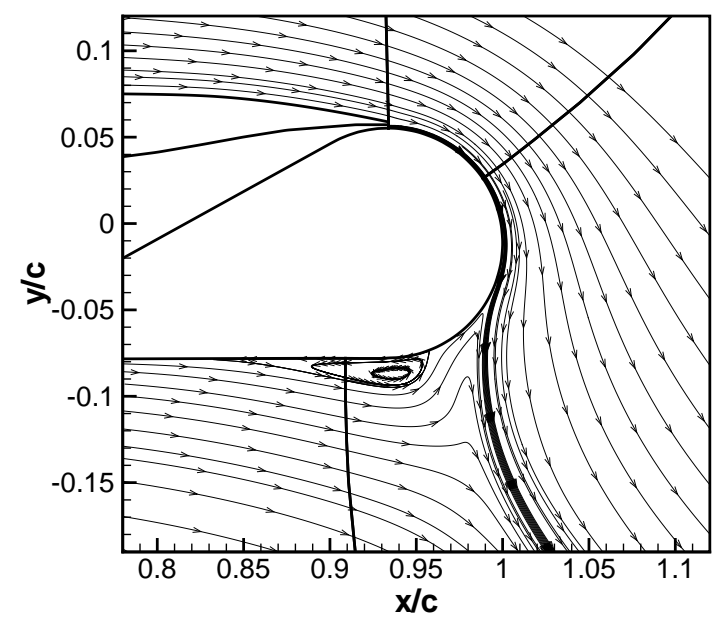

(a)

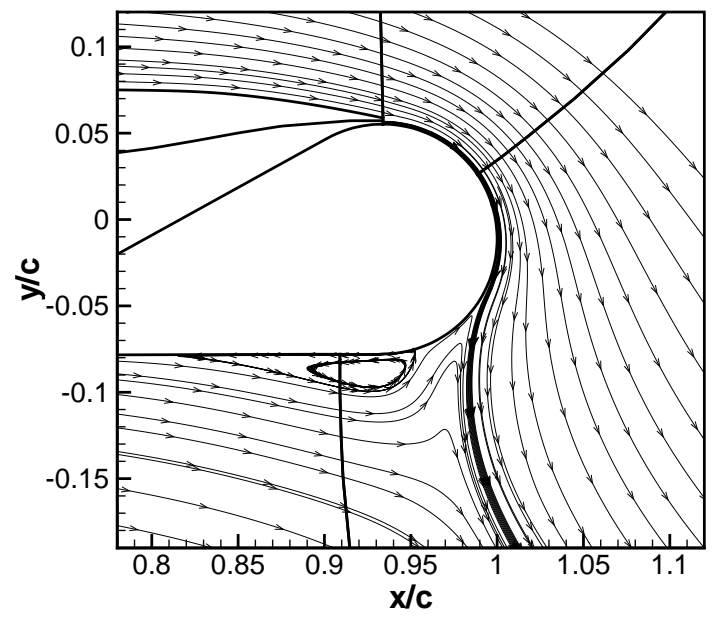

(b)

Figure 19. Lockheed case streamlines, computations with SST model, $C_{\mu}=0.10:$ (a) grid 5: fine, (b) grid 7: medium, time accurate. 

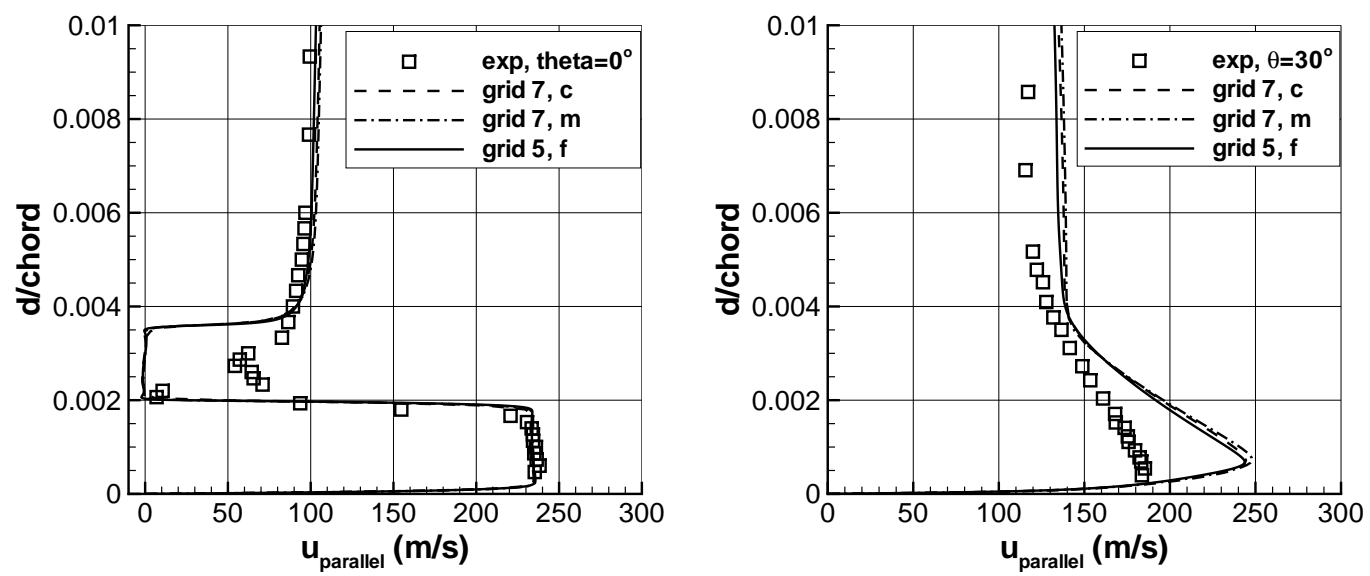

Figure 20a. Lockheed case velocity profiles, $C_{\mu}=0.10$, SST model, grid 7: time accurate, $\theta=0,30$ degrees.
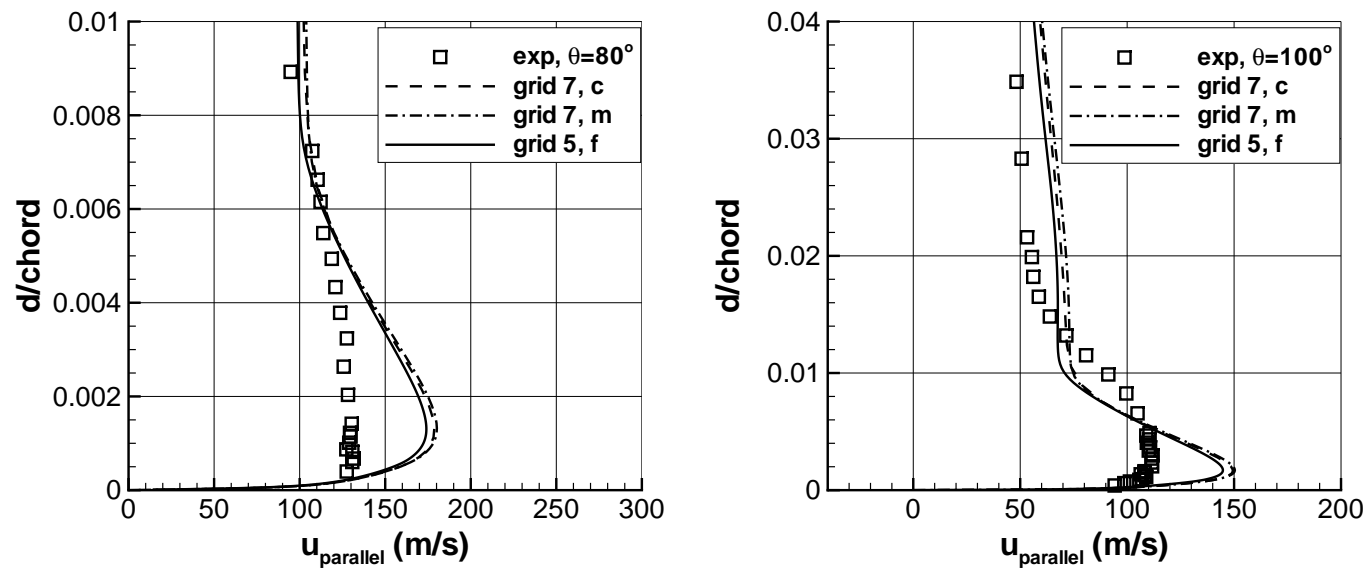

Figure 20b. Lockheed case velocity profiles, $C_{\mu}=0.10$, SST model, grid 7: time accurate, $\theta=80,100$ degrees.
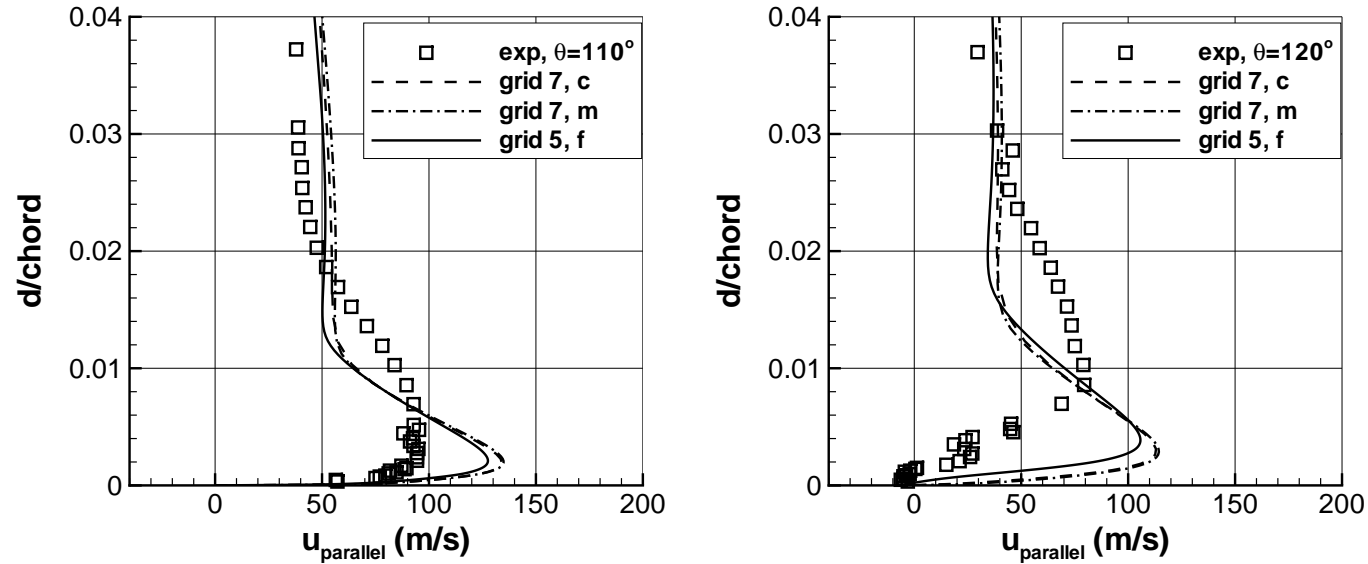

Figure 20c. Lockheed case velocity profiles, $C_{\mu}=0.10$, SST model, grid 7: time accurate, $\theta=110,120$ degrees. 

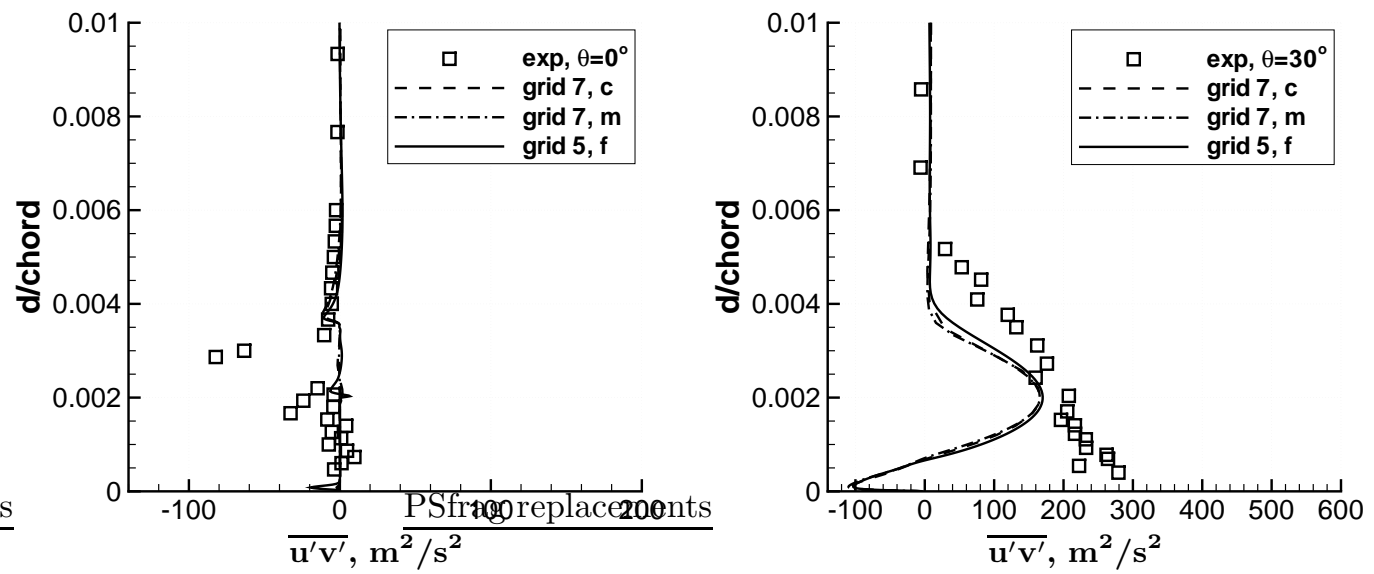

Figure 21a. Lockheed case shear stress profiles, $C_{\mu}=0.10$, SST model, grid 7: time accurate, $\theta=0,30$ degrees.
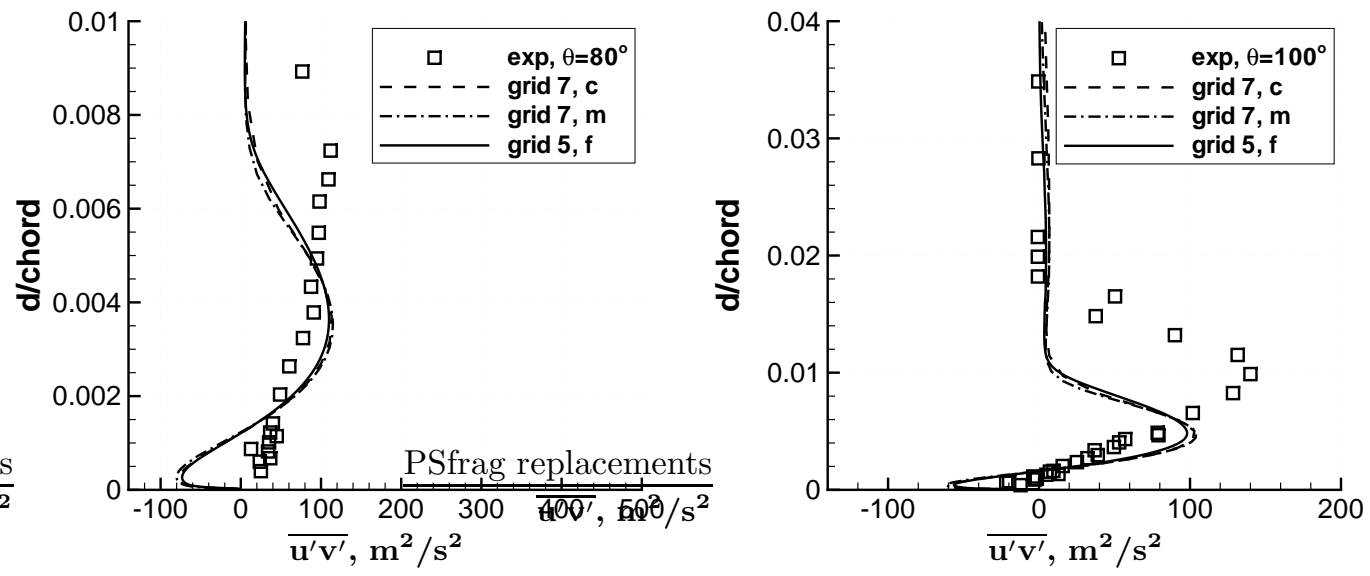

Figure 21b. Lockheed case shear stress profiles, $C_{\mu}=0.10$, SST model, grid 7: time accurate, $\theta=80,100$ degrees.
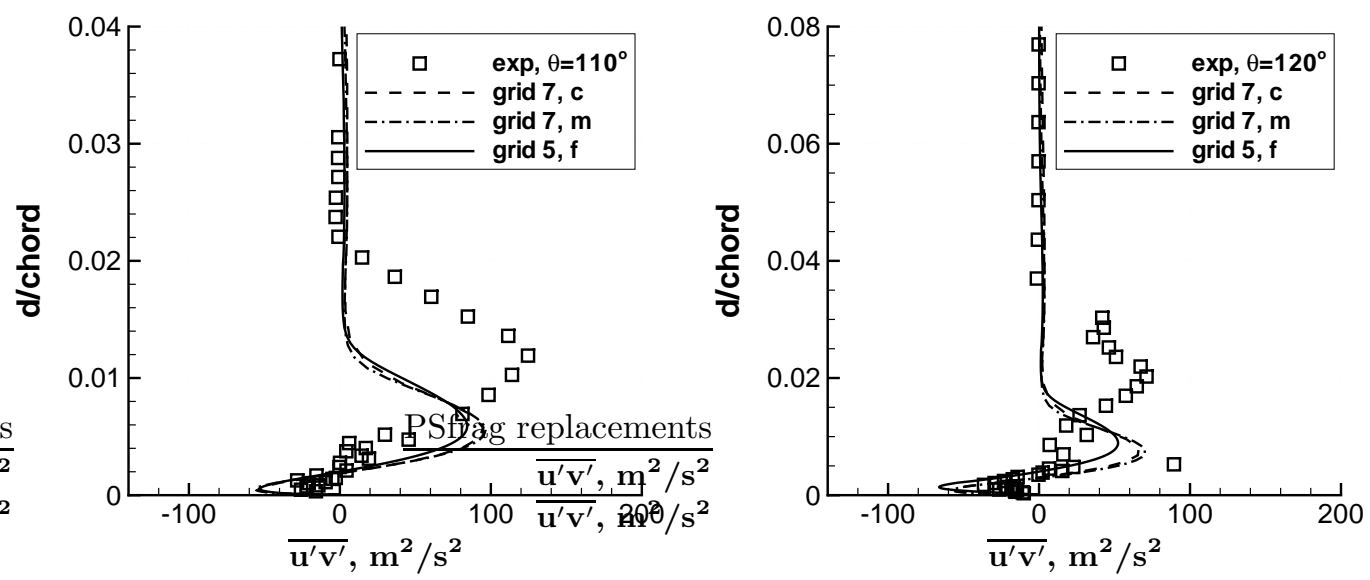

Figure 21c. Lockheed case shear stress profiles, $C_{\mu}=0.10$, SST model, grid 7: time accurate, $\theta=110,120$ degrees. 


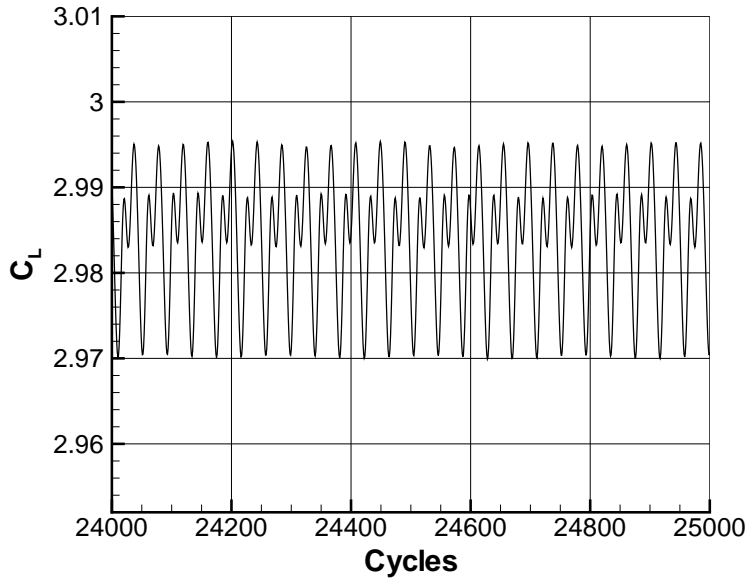

Figure 22. GACC case lift coefficient history, medium grid.

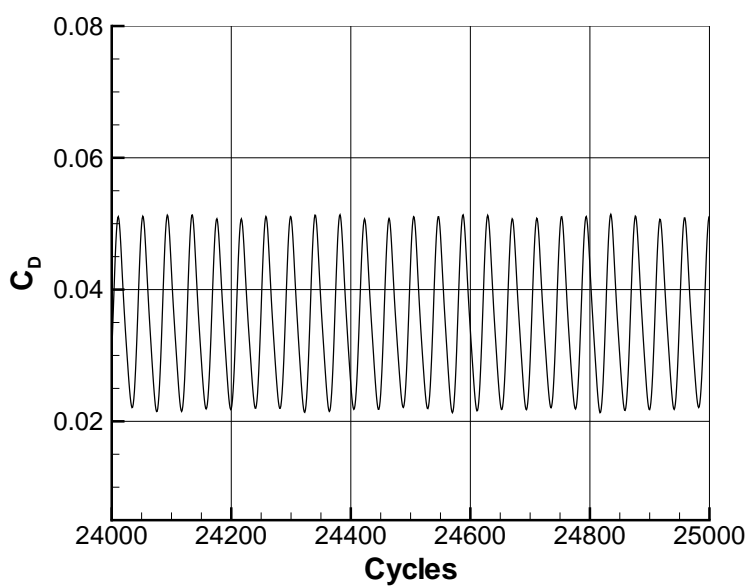

Figure 23. GACC case drag coefficient history, medium grid.

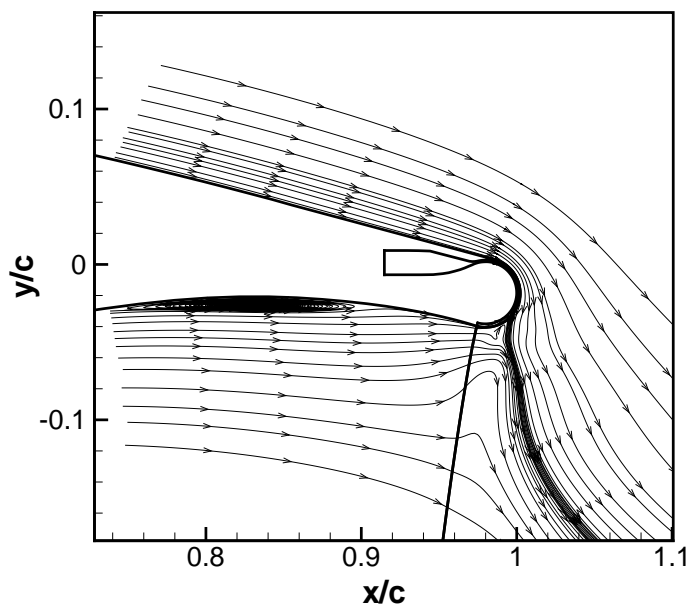

Figure 24. GACC case streamlines at trailing edge, medium grid, SST model.

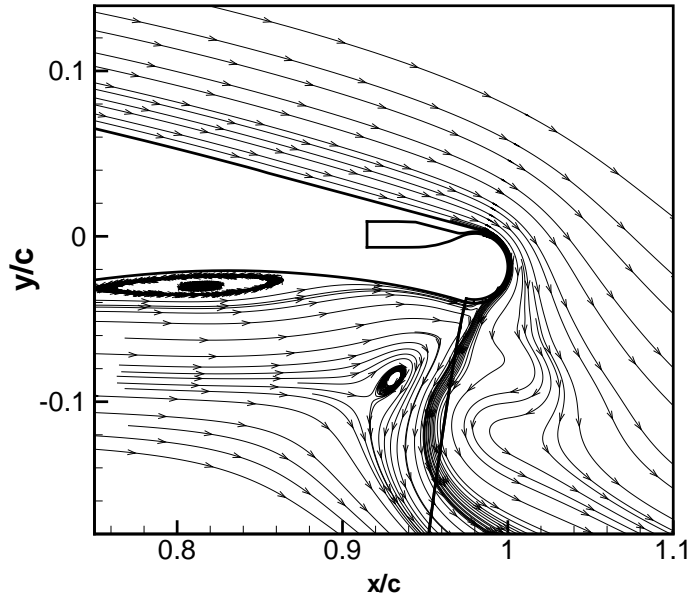

Figure 25. GACC case streamlines at trailing edge, fine grid, SST model.

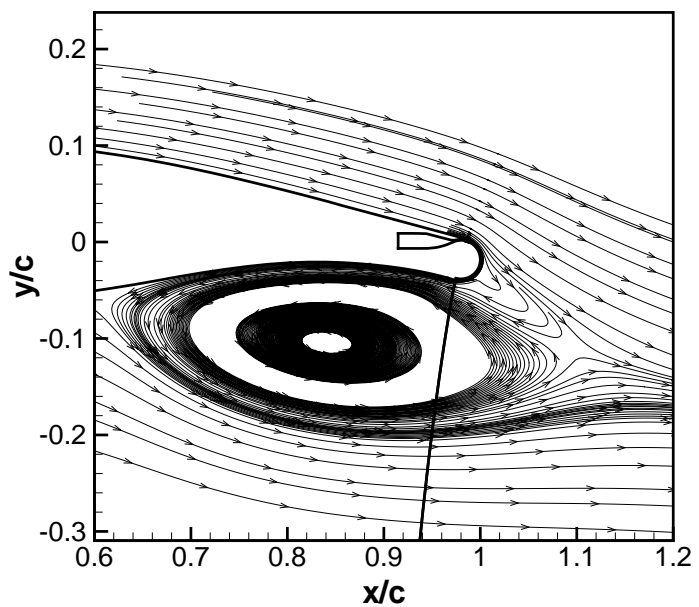

Figure 26. GACC case streamlines at trailing edge, medium grid, SARC model. 


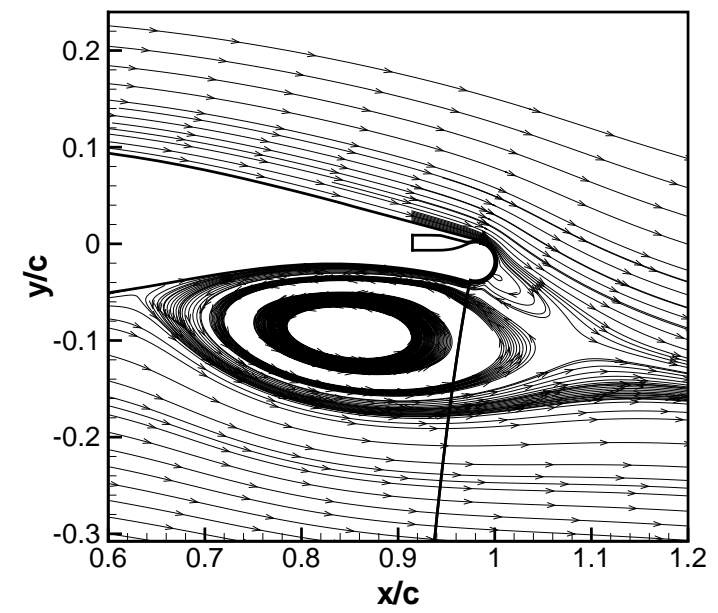

Figure 27. GACC case streamlines at trailing edge, medium grid, SA model.

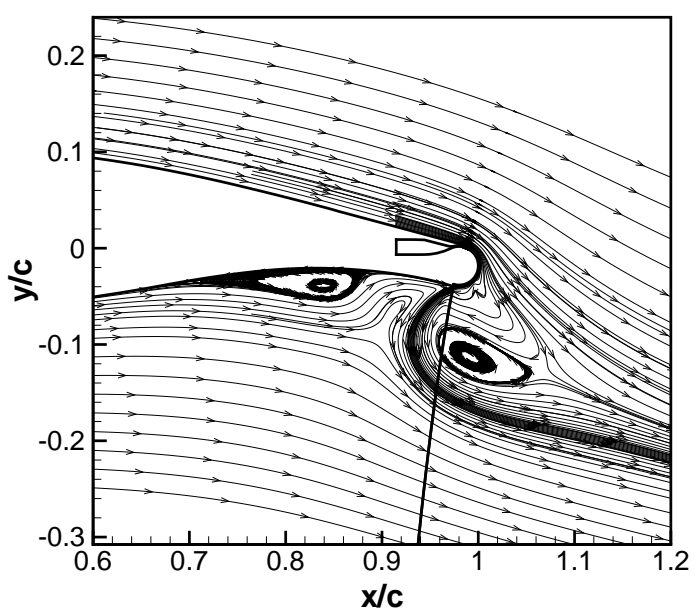

Figure 28. GACC case streamlines at trailing edge, fine grid, SA model.

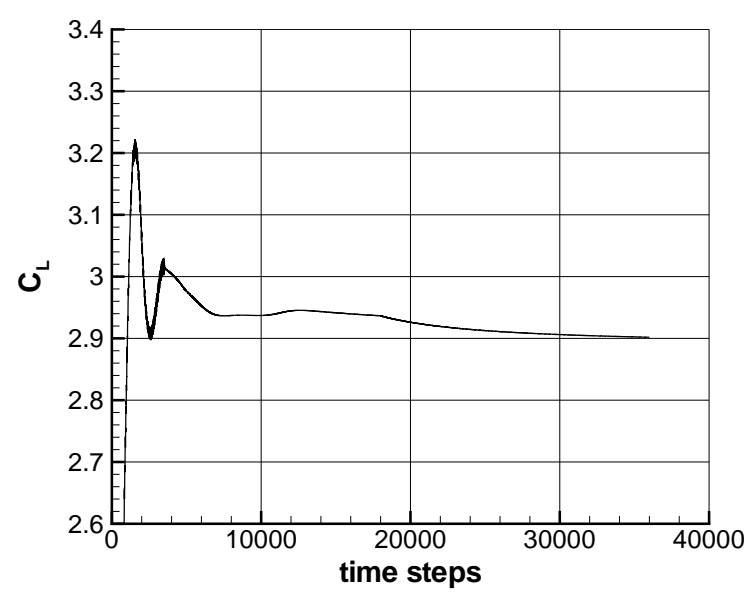

Figure 29. GACC case lift coefficient history, medium grid, time accurate.

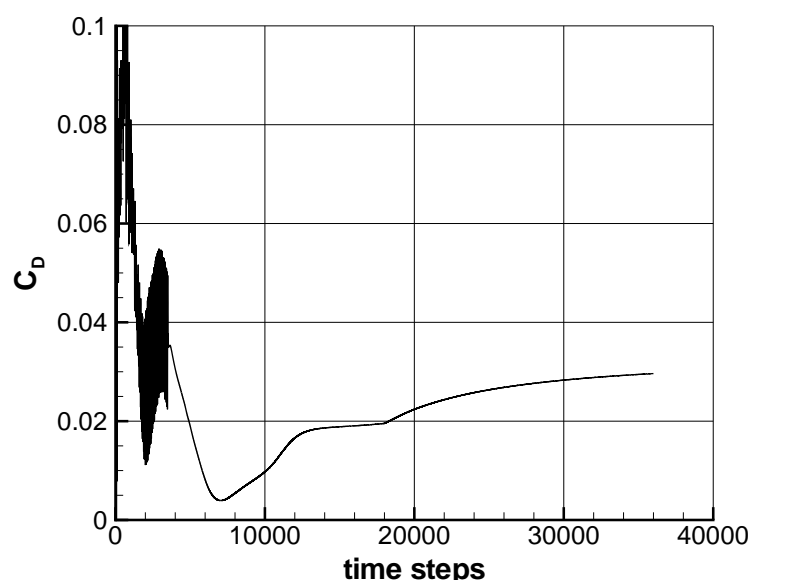

Figure 30. GACC case drag coefficient history, medium grid, time accurate.

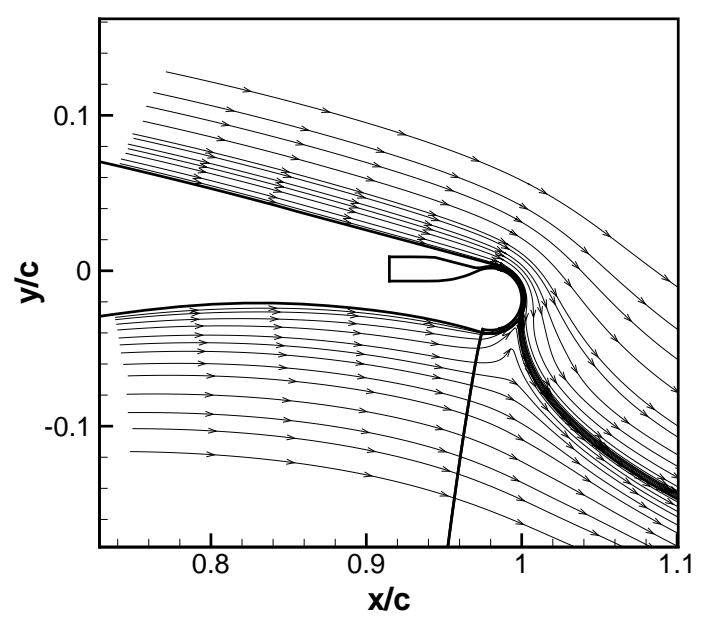

Figure 31. GACC case streamlines at trailing edge, medium grid, SST model, time accurate. 


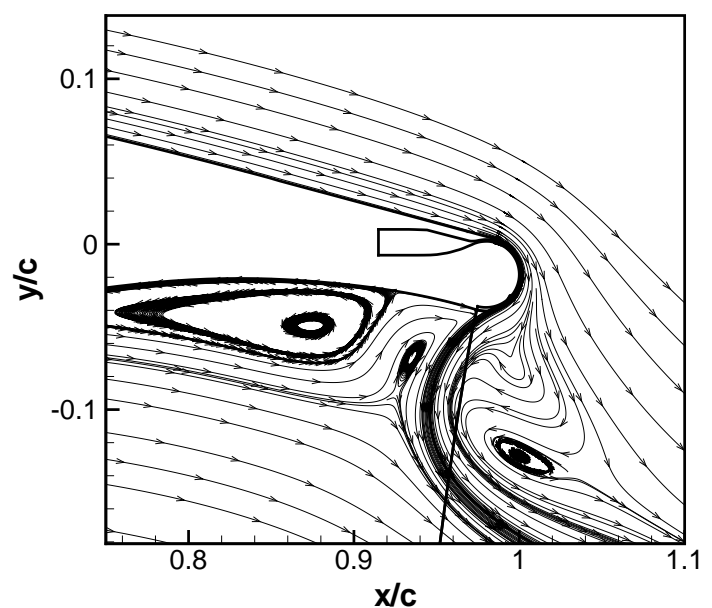

Figure 32. GACC case streamlines at trailing edge, fine grid, SST model, time accurate.

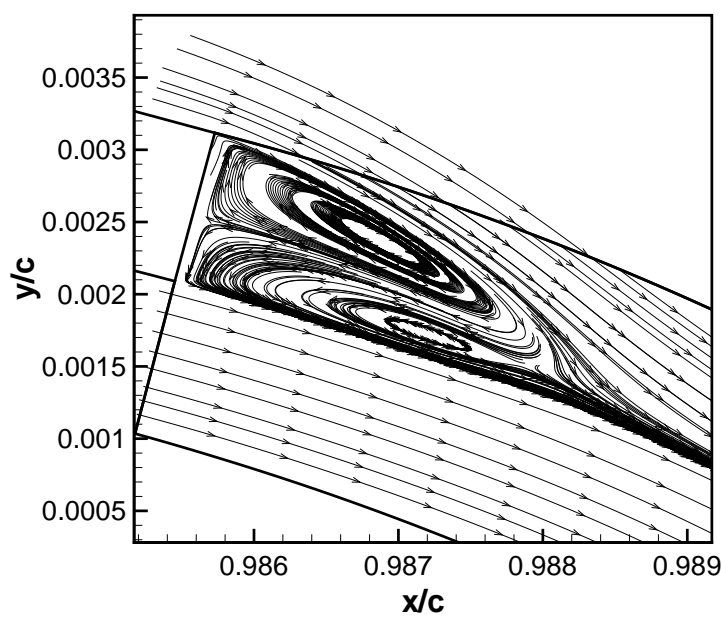

Figure 33. GACC case streamlines at lip of jet slot.

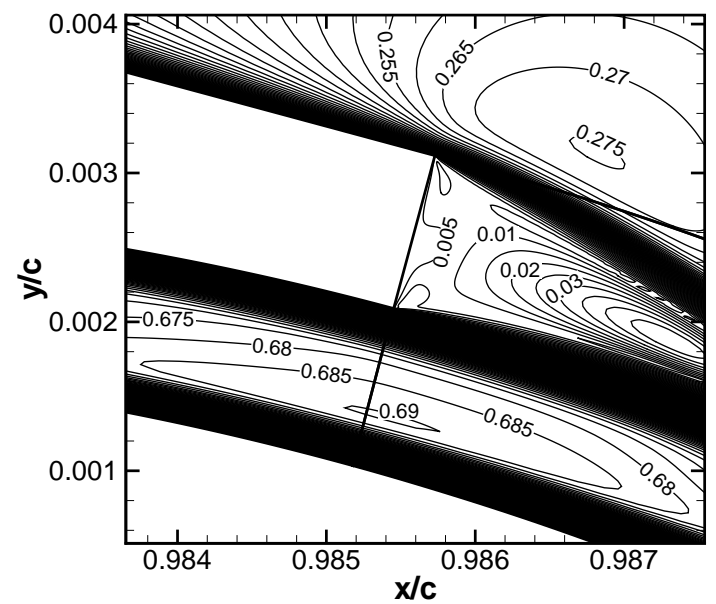

Figure 34. GACC case Mach contours near jet slot exit.

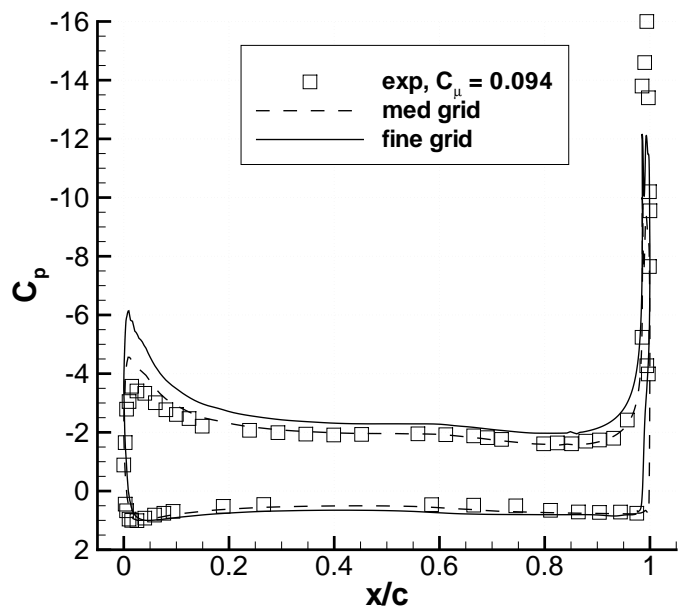

Figure 35. GACC case surface presure distributions, computations with $C_{\mu}=0.093$, time accurate.

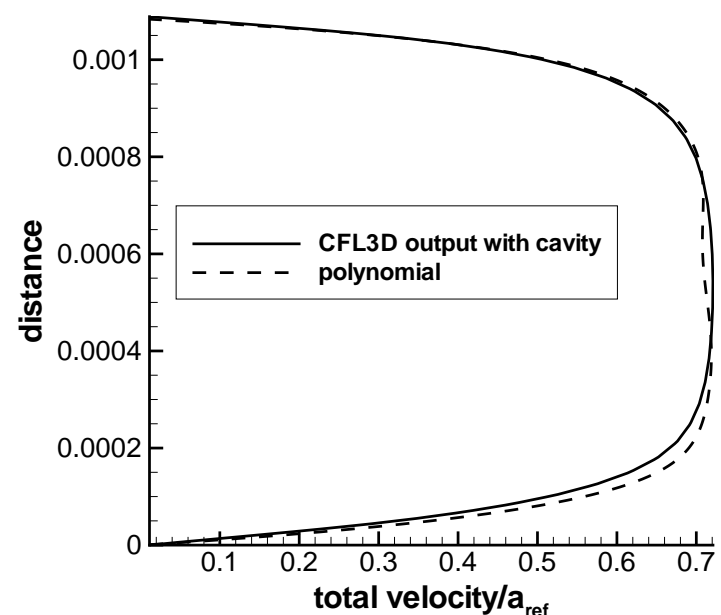

Figure 36. GACC case total velocity profiles at slot exit.

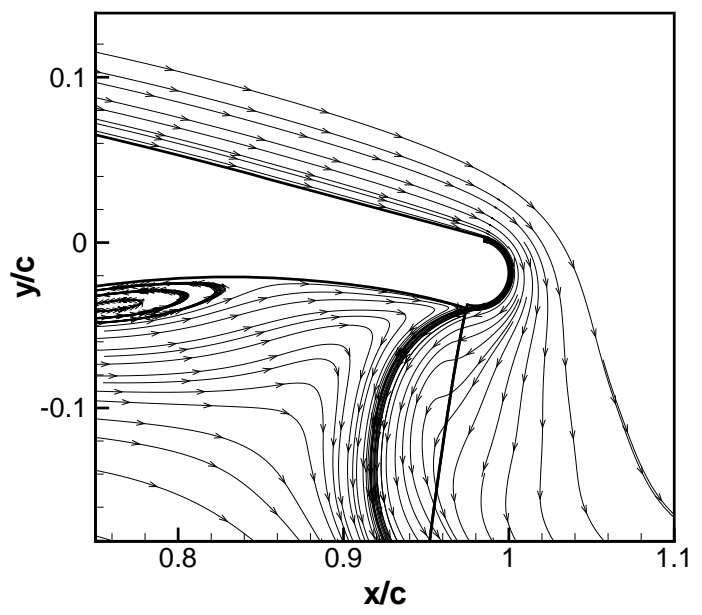

Figure 37. GACC case streamlines at trailing edge, medium grid, SST model, time accurate, no plenum. 